\title{
Finiteness results for K3 surfaces over arbitrary fields
}

\author{
Martin Bright ${ }^{1} \cdot$ Adam Logan $^{2,3} \cdot$ Ronald van Luijk $^{1}$
}

Received: 19 October 2018 / Revised: 5 March 2019 / Accepted: 18 April 2019 / Published online: 6 May 2019 (c) The Author(s) 2019

\begin{abstract}
Over an algebraically closed field, various finiteness results are known regarding the automorphism group of a K3 surface and the action of the automorphisms on the Picard lattice. We formulate and prove versions of these results over arbitrary base fields, and give examples illustrating how behaviour can differ from the algebraically closed case.
\end{abstract}

Keywords K3 surfaces · Automorphism groups · Picard groups · Non-algebraically closed fields

Mathematics Subject Classification 14J28 $\cdot 14 \mathrm{~J} 50 \cdot 14 \mathrm{G} 27$

\section{Introduction}

The geometry of K3 surfaces over the complex numbers has a long history, with many results known about the cohomology, the Picard group, and the automorphism group of an algebraic K3 surface, and how these objects interact. Such results over the complex numbers carry over to other algebraically closed fields of characteristic zero, and

The second author would like to thank the Tutte Institute for Mathematics and Computation for its partial support for a visit to the University of Leiden during which much of this research was done.

$凶$ Martin Bright

m.j.bright@math.leidenuniv.nl

Adam Logan

adam.m.logan@gmail.com

Ronald van Luijk

rvl@math.leidenuniv.nl

1 Mathematisch Instituut, Niels Bohrweg 1, 2333 CA Leiden, The Netherlands

2 The Tutte Institute for Mathematics and Computation, P.O. Box 9703, Terminal, Ottawa, ON K1G 3Z4, Canada

3 School of Mathematics and Statistics, 4302 Herzberg Laboratories, Carleton University, 1125 Colonel By Drive, Ottawa, ON K1S 5B6, Canada 
similar results are also known over algebraically closed fields of other characteristics. For a comprehensive treatment of the geometry of K3 surfaces, we refer the reader to the lecture notes of Huybrechts [12]. Much of the theory we use was originally developed by Nikulin [15].

K3 surfaces are also interesting from an arithmetic point of view, with much recent work on understanding the rational points, curves, Brauer groups and other invariants of K3 surfaces over number fields. In this article, we investigate the extent to which some standard finiteness results for K3 surfaces over algebraically closed fields remain true over more general base fields. In particular, we show how to define the correct analogue of the Weyl group, and give an explicit description of it. This allows us to formulate and prove finiteness theorems over arbitrary fields, modelled on those already known over algebraically closed fields. The tools we use include representability of the Picard and automorphism schemes, classification of transitive group actions on Coxeter-Dynkin diagrams, and an explicit description of the walls of the ample cone. We follow these theoretical results with several detailed examples, showing how the relationship between the Picard group and the automorphism group can be different from the geometric case. We end by proving that a surface over $\mathbb{Q}$ of the form $x^{4}-y^{4}=c\left(z^{4}-w^{4}\right)$ has finite automorphism group for $c \in \mathbb{Q}^{*}$ that is not in the subgroup generated by squares together with $-1,2$.

The specific finiteness results we address go back to Sterk [20]. To state them, we need some definitions; we follow the notation of [12]. In this article, by a K3 surface we will always mean an algebraic $\mathrm{K} 3$ surface, which is therefore projective.

Let $k$ be a field, and let $X$ be a K3 surface over $k$. Denote the group of isometries of Pic $X$ by $\mathrm{O}(\operatorname{Pic} X)$. Reflection in any (-2)-class in Pic $X$ defines an isometry of Pic $X$, and we define the Weyl group $\mathrm{W}(\operatorname{Pic} X) \subset \mathrm{O}(\operatorname{Pic} X)$ to be the subgroup generated by these reflections.

We recall the definitions of the positive, ample and nef cones associated to the $\mathrm{K} 3$ surface $X$; see also [12, Chapter 8]. Let $(\mathrm{Pic} X)_{\mathbb{R}}$ denote the real vector space $(\operatorname{Pic} X) \otimes_{\mathbb{Z}} \mathbb{R}$. By the Hodge index theorem, the intersection product on $(\operatorname{Pic} X)_{\mathbb{R}}$ has signature $(1, \rho-1)$; so the set $\left\{\alpha \in(\operatorname{Pic} X)_{\mathbb{R}} \mid \alpha^{2}>0\right\}$ consists of two connected components. The positive cone $\mathrm{C}_{X} \subset(\operatorname{Pic} X)_{\mathbb{R}}$ is the connected component containing all the ample classes.

The ample cone $\operatorname{Amp}(X)$ is the cone in $(\operatorname{Pic} X)_{\mathbb{R}}$ generated by all classes of ample line bundles. The nef cone $\operatorname{Nef}(X)$ is defined as

$$
\operatorname{Nef}(X)=\left\{\alpha \in(\operatorname{Pic} X)_{\mathbb{R}} \mid \alpha \cdot C \geqslant 0 \text { for all curves } C \subset X\right\} .
$$

Finally, we define $\operatorname{Nef}^{\mathrm{e}}(X)$ to be the real convex hull of $\operatorname{Nef}(X) \cap \operatorname{Pic} X$. An application of the criterion of Nakai-Moishezon-Kleiman shows that $\operatorname{Amp}(X)$ is the interior of $\operatorname{Nef}(X)$ and $\operatorname{Nef}(X)$ is the closure of $\operatorname{Amp}(X)$ : see [12, Corollary 8.1.4].

The following finiteness theorems are due to Sterk [20] for $k=\mathbb{C}$ and to Lieblich and Maulik [13] when $k$ has positive characteristic not equal to 2. As in Huybrechts [12, Chapter 8], a fundamental domain for the action of a discrete group $G$ acting continuously on a topological manifold $M$ is defined as the closure $\bar{U}$ of an open subset $U \subset M$ such that $M=\bigcup_{g \in G} g \bar{U}$ and such that for $g \neq h \in G$ the intersection $g \bar{U} \cap h \bar{U}$ does not contain interior points of $g U$ or $h U$. 
Theorem 1.1 Let $k=\bar{k}$ be an algebraically closed field of characteristic not equal to 2 , and let $X$ be a $K 3$ surface over $k$.

(1) ([12, Corollary 8.2.11]) The cone $\operatorname{Nef}(X) \cap \mathcal{C}_{X}$ is a fundamental domain for the action of $\mathrm{W}(\mathrm{Pic} X) \subset \mathrm{O}(\mathrm{Pic} X)$ on the positive cone $\mathcal{C}_{X}$.

(2) ([12, Theorem 15.2.6], [13, Proposition 5.2]) The subgroup $\mathrm{W}(\operatorname{Pic} X)$ is normal in $\mathrm{O}(\operatorname{Pic} X)$; the natural map Aut $X \rightarrow \mathrm{O}(\operatorname{Pic} X) / \mathrm{W}(\operatorname{Pic} X)$ has finite kernel and image of finite index.

(3) ([12, Theorem 8.4.2]) The action of Aut $X$ on $\mathrm{Nef}^{\mathrm{e}} X$ admits a rational polyhedral fundamental domain.

(4) ([12, Corollary 8.4.6]) The set of orbits under Aut $X$ of (-2)-curves on $X$ is finite. More generally, for any $d$ there are only finitely many orbits under Aut $X$ of classes of irreducible curves of self-intersection $2 d$.

In Sect. 3 we will prove analogues of the various statements of Theorem 1.1 when $k$ is replaced by an arbitrary base field of characteristic different from 2 .

A consequence of Theorem 1.1(2) is that the finiteness of Aut $X$ depends only on Pic $X$. Those possible Picard lattices for which the quotient $\mathrm{O}(\mathrm{Pic} X) / \mathrm{W}(\operatorname{Pic} X)$ is finite have been classified in [14]. Over an arbitrary base field we will see that, instead of using the Weyl group W(Pic $X)$, we must use the Galois-invariant part of the geometric Weyl group. This means that the finiteness of Aut $X$ is no longer determined purely by the Picard lattice Pic $X$; rather, it depends on the geometric Picard lattice together with the Galois action. In Sect. 4, we give several examples that illustrate this difference to the classical case.

\section{Lemmas on lattices}

In this section we will study lattices with the action of a group. Given a lattice $\Lambda$ with the action of a finite group $H$, we consider the group of automorphisms that commute with $H$, and the group of automorphisms that preserve the sublattice fixed by $H$.

Definition 2.1 A lattice $\Lambda$ is a free abelian group of finite rank with a non-degenerate integer-valued symmetric bilinear form. If the form is (positive or negative) definite, we likewise refer to $\Lambda$ as definite. The group of automorphisms of $\Lambda$ preserving the form is denoted $\mathrm{O}(\Lambda)$. A sublattice of $\Lambda$ is a subgroup on which the restriction of the form is non-degenerate. Given a sublattice $M \subseteq \Lambda$, we use $\mathrm{O}(\Lambda, M)$ for the subgroup of $\mathrm{O}(\Lambda)$ fixing $M$ as a set. For a subgroup $H \subseteq \mathrm{O}(\Lambda)$, let $\Lambda^{H}$ be the subgroup of $\Lambda$ consisting of the elements fixed by every element of $H$ (note that according to our conventions $\Lambda^{H}$ may not be a lattice, because the quadratic form on $\Lambda$ may be degenerate when restricted to $\Lambda^{H}$ ). The vector space $\Lambda \otimes_{\mathbb{Z}} \mathbb{Q}$ will be denoted as $\Lambda_{\mathbb{Q}}$.

Our goal is to prove the following proposition.

Proposition 2.2 Let $\Lambda$ be a lattice and $H \subseteq \mathrm{O}(\Lambda)$ a subgroup such that $M=\Lambda^{H}$ is a lattice. Then the following hold:

(1) the natural map $\mathrm{O}(\Lambda, M) \rightarrow \mathrm{O}(M)$ has image of finite index;

(2) suppose that $M^{\perp}$ is definite. Then $\mathrm{O}(\Lambda, M) \rightarrow \mathrm{O}(M)$ has finite kernel, and the centralizer $Z_{\mathrm{O}(\Lambda)} H$ is a finite-index subgroup of $\mathrm{O}(\Lambda, M)$. 
Our interest in this situation arises from the geometry of $\mathrm{K} 3$ surfaces. Let $X$ be a projective $\mathrm{K} 3$ surface defined over a field $F$, and let $K / F$ be a Galois extension. Let $\Lambda=\operatorname{Pic} X_{K}$ with the intersection pairing, and let $H$ be the image of $\operatorname{Gal}(K / F)$ in $\mathrm{O}(\Lambda)$. If $X$ has a rational point over $F$, we have Pic $X_{F}=\Lambda^{H}$. (See Sect. 3 for more details. This statement holds slightly more generally: for example, if $F$ is a number field and $X$ has points everywhere locally over $F$.) The Hodge index theorem states that $\Lambda$ has signature $(1, n)$ and $\Lambda^{H}$ has signature $(1, m)$ : therefore $M^{\perp}$ is definite.

Before giving the proof we first collect a few helpful statements, which are probably well known.

Lemma 2.3 Let $\Lambda$ be a lattice and $M$ a sublattice. Let $H$ be a subgroup of $\mathrm{O}(\Lambda)$ such that $\Lambda^{H}$ is a lattice. For groups $G^{\prime} \subset G$, let $Z_{G}\left(G^{\prime}\right)$ denote the centralizer of $G^{\prime}$ in $G$.

(1) $M^{\perp}$ is a sublattice of $\Lambda$.

(2) There is a natural injection $d: \mathrm{O}(\Lambda, M) \hookrightarrow \mathrm{O}(M) \oplus \mathrm{O}\left(M^{\perp}\right)$ with image of finite index.

(3) $Z_{\mathrm{O}(\Lambda)} H$ is contained in $\mathrm{O}\left(\Lambda, \Lambda^{H}\right)$.

(4) The kernel of the map $\mathrm{O}\left(\Lambda, \Lambda^{H}\right) \rightarrow \mathrm{O}\left(\Lambda^{H}\right)$ is contained in $Z_{\mathrm{O}(\Lambda)} H$.

Proof To prove (1), we just have to prove that the pairing on the subspace $M_{\mathbb{Q}}^{\perp} \subset \Lambda_{\mathbb{Q}}$ is non-degenerate; this is [7, Satz 1.2].

Let $\phi \in \mathrm{O}(\Lambda, M)$. By definition $\phi$ restricts to an endomorphism of $M$. Let $S_{M}$ be the saturation of $M$ in $\Lambda$. Clearly $\phi\left(S_{M}\right) \subseteq S_{M}$. Now, $\phi^{-1}\left(S_{M}\right)$ has the same rank as $S_{M}$ and contains $S_{M}$, so it is equal to $S_{M}$. So if $\phi\left(S_{M}\right) \neq S_{M}$, then the image of $\phi$ does not contain $S_{M}$, contradicting the hypothesis that $\phi$ is an automorphism of $\Lambda$. Since $M$ is a subgroup of finite index of $S_{M}$, this implies that \# $\left(S_{M} / M\right)=\#\left(S_{M} / \phi(M)\right)$. But $\phi(M) \subseteq M$, so it follows that $\phi(M)=M$. Thus there is a map $\mathrm{O}(\Lambda, M) \rightarrow \mathrm{O}(M)$. Now, if $\phi \in \mathrm{O}(\Lambda, M)$ and $y \in M^{\perp}$, then $m \cdot \phi(y)=\phi^{-1}(m) \cdot y=0$ for all $m \in M$, so $\phi(y) \in M^{\perp}$, and we get a map $\mathrm{O}(\Lambda, M) \rightarrow \mathrm{O}\left(M^{\perp}\right)$ in the same way. Combining these two maps gives a map $d: \mathrm{O}(\Lambda, M) \rightarrow \mathrm{O}(M) \oplus \mathrm{O}\left(M^{\perp}\right)$.

If $d(\phi)=1$, then $d$ is the identity on $M \oplus M^{\perp}$, which is a subgroup of $\Lambda$ of finite index. Because $\Lambda$ is torsion-free, this forces $\phi$ to be the identity. To show that im $d$ has finite image in $\mathrm{O}(M) \oplus \mathrm{O}\left(M^{\perp}\right)$, let $n$ be the smallest positive integer such that $n \Lambda \subseteq M \oplus M^{\perp}$, and let $k=\left[M \oplus M^{\perp}: n \Lambda\right]$. Every element of $\mathrm{O}(M) \oplus \mathrm{O}\left(M^{\perp}\right)$ that fixes $n \Lambda$ as a set is in the image of $d$, because the induced automorphism of $n \Lambda$ extends to an automorphism of $\Lambda$ with the same action on $M \oplus M^{\perp}$. Since there are only finitely many subgroups of index $k$ in $M \oplus M^{\perp}$, the stabilizer of $n \Lambda$ is of finite index, and we have proved (2).

To prove (3), let $\phi \in Z_{\mathrm{O}(\Lambda)} H$, and let $m \in \Lambda^{H}$ and $h \in H$. Then $h(m)=m$ and $\phi \circ h=h \circ \phi$. So $h(\phi(m))=\phi(h(m))=\phi(m)$, establishing that $\phi(m) \in \Lambda^{H}$.

Finally we prove (4). Choose $\phi$ in the kernel and $h \in H$, and let $x \in \Lambda$. We will view $\phi$ and $h$ as automorphisms of $\Lambda_{\mathbb{Q}}$. In $\Lambda_{\mathbb{Q}}$ we may write $x=x_{1}+x_{2}$, where $x_{1} \in \Lambda_{\mathbb{Q}}^{H}$ and $x_{2} \in\left(\Lambda_{\mathbb{Q}}^{H}\right)^{\perp}$. Then $h(\phi(x))=h\left(\phi\left(x_{1}+x_{2}\right)\right)=h\left(\phi\left(x_{1}\right)\right)+h\left(\phi\left(x_{2}\right)\right)$. However, $\phi\left(x_{1}\right) \in \Lambda_{\mathbb{Q}}^{H}$, so $h\left(\phi\left(x_{1}\right)\right)=\phi\left(x_{1}\right)$, and $\phi$ is in the kernel of the map to $\mathrm{O}\left(\Lambda^{H^{\perp}}\right)$, so $\phi\left(x_{2}\right)=x_{2}$. It follows that $h(\phi(x))=\phi\left(x_{1}\right)+h\left(x_{2}\right)$. Similarly, $\phi(h(x))=\phi\left(h\left(x_{1}+x_{2}\right)\right)=\phi\left(h\left(x_{1}\right)\right)+\phi\left(h\left(x_{2}\right)\right)=\phi\left(x_{1}\right)+h\left(x_{2}\right)=h(\phi(x))$, establishing that $\phi$ commutes with $h$. 
Proof of Proposition 2.2 The map $\mathrm{O}(\Lambda, M) \rightarrow \mathrm{O}(M)$ is a composition $\mathrm{O}(\Lambda, M) \rightarrow$ $\mathrm{O}(M) \oplus \mathrm{O}\left(M^{\perp}\right) \rightarrow \mathrm{O}(M)$. In part (2) of the lemma just proved we showed that the first map has image of finite index. The second map is surjective, so the composition has image of finite index as well.

We now suppose that $M^{\perp}$ is definite to prove the second statement. Then $\mathrm{O}\left(M^{\perp}\right)$ is finite, so $\mathrm{O}(\Lambda, M) \rightarrow \mathrm{O}(M)$ is a composition of an injective map with a map with finite kernel and so its kernel is finite. Let $K=\operatorname{ker}\left(\mathrm{O}(\Lambda, M) \rightarrow \mathrm{O}\left(M^{\perp}\right)\right)$. Then $Z_{\mathrm{O}(\Lambda)} H / K$ has finite index in $\mathrm{O}(\Lambda, M) / K$, because both inject into the finite group $\mathrm{O}\left(M^{\perp}\right)$. Therefore $Z_{\mathrm{O}(\Lambda)} H$ has finite index in $\mathrm{O}(\Lambda, M)$ too.

\section{Finiteness results for K3 surfaces}

In this section we formulate and prove analogues of the statements of Theorem 1.1 when $k$ is an arbitrary field. We first look at the case of $k$ separably closed, which is straightforward.

Lemma 3.1 Let $k=k^{\mathrm{s}}$ be a separably closed field, and let $\bar{k}$ be an algebraic closure of $k$. Let $X$ be a K3 surface over $k$, and let $\bar{X}$ be the base change of $X$ to $\bar{k}$. Then the natural maps $\operatorname{Pic} X \rightarrow \operatorname{Pic} \bar{X}$ and Aut $X \rightarrow$ Aut $\bar{X}$ are isomorphisms.

Proof As $X$ is projective, the Picard scheme Pic $\mathbf{P}_{X / k}$ exists, is separated and locally

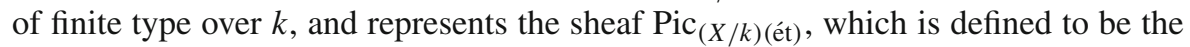
sheafification on the big étale site over $k$ of the presheaf

$$
T \mapsto \operatorname{Pic}\left(X \times{ }_{k} T\right) / \operatorname{Pic} T
$$

(see [9, Theorem 9.4.8]). In particular, because $k$ and $\bar{k}$ are both separably closed, we have $\operatorname{Pic}_{X / k}(k)=\operatorname{Pic} X$ and $\operatorname{Pic}_{X / k}(\bar{k})=\operatorname{Pic} \bar{X}$. From the triviality of $\mathrm{H}^{1}\left(X, \mathcal{O}_{X}\right)$ it follows from [9, Theorem 9.5.11] that $\mathbf{P i c}_{X / k}$ is étale over $k$. So every $\bar{k}$-point of Pic $_{X / k}$ is defined over $k$, and Pic $X \rightarrow \operatorname{Pic} \bar{X}$ is an isomorphism.

The functor taking a $k$-scheme $S$ to the group Aut $\left(X \times_{k} S\right)$ is represented by a scheme Aut ${ }_{X / k}$ : see [9, Theorem 5.23]. A standard argument in deformation theory shows that the tangent space at the identity element is isomorphic to $\mathrm{H}^{0}\left(X, T_{X}\right)$, where $T_{X}$ denotes the tangent sheaf on $X$. Indeed, an element of the tangent space is given by a morphism $S=\operatorname{Spec} k[\varepsilon] /\left(\varepsilon^{2}\right) \rightarrow$ Aut $_{X / k}$ extending the morphism sending Spec $k$ to the identity automorphism. Such a morphism corresponds to an automorphism of $X \times{ }_{k} S$ restricting to the identity on the central fibre. By [9, Theorem 8.5.9], the set of these morphisms forms an affine space under $\mathrm{H}^{0}\left(X, T_{X}\right)$. In our case, the group $\mathrm{H}^{0}\left(X, T_{X}\right)$ is zero [12, Theorem 9.5.1], so the scheme Aut ${ }_{X / k}$ is étale over $k$, and Aut $X \rightarrow$ Aut $\bar{X}$ is an isomorphism.

Corollary 3.2 In the situation of Lemma 3.1, every (-2)-curve on $\bar{X}$ is defined over $k$.

Proof Let $\bar{C}$ be a $(-2)$-curve on $\bar{X}$. Then Lemma 3.1 shows that there is a line bundle $L$ on $X$ whose base change to $\bar{X}$ is isomorphic to $\mathcal{O}_{\bar{X}}(\bar{C})$. The Riemann-Roch theorem and flat base change give $h^{0}(X, L)=h^{0}\left(\bar{X}, \mathcal{O}_{\bar{X}}(\bar{C})\right)=1$. So all non-zero sections of 
$L$ cut out the same divisor $C \subset X$ and the base change of $C$ to $\bar{X}$ must coincide with $\bar{C}$. In other words, $\bar{C}$ is defined over $k$.

We now pass to the case of a general field. Let $k$ be a field; fix an algebraic closure $\bar{k}$ of $k$, and let $k^{\mathrm{s}}$ be the separable closure of $k$ in $\bar{k}$. Let $X$ be a K3 surface over $k$, and let $X^{\mathrm{s}}$ and $\bar{X}$ denote the base changes of $X$ to $k^{\mathrm{s}}$ and $\bar{k}$, respectively. Write $\Gamma_{k}=\operatorname{Gal}\left(k^{\mathrm{s}} / k\right)$.

The group $\Gamma_{k}$ acts on Pic $X^{\mathrm{s}}$ preserving intersection numbers, giving a representation $\Gamma_{k} \rightarrow \mathrm{O}\left(\operatorname{Pic} X^{\mathrm{s}}\right)$. Let $\Gamma_{k}$ act on $\mathrm{O}\left(\operatorname{Pic} X^{\mathrm{s}}\right)$ by conjugation, that is, so that $(\sigma f)(x)=\sigma\left(f\left(\sigma^{-1} x\right)\right)$ for all $x \in \operatorname{Pic} X^{\mathrm{s}}$. For a (-2)-class $\alpha \in \operatorname{Pic} X^{\mathrm{s}}$, denote the reflection in $\alpha$ by $r_{\alpha}$; then we have $\left({ }^{\sigma} r_{\alpha}\right)=r_{\sigma \alpha}$. So the action of $\Gamma_{k}$ on $\mathrm{O}\left(\operatorname{Pic} X^{\mathrm{s}}\right)$ restricts to an action on $\mathrm{W}\left(\operatorname{Pic} X^{\mathrm{s}}\right)$.

Definition 3.3 Define $R_{X}$ to be the group $\mathrm{W}\left(\operatorname{Pic} X^{\mathrm{s}}\right)^{\Gamma_{k}}$.

Recall that Pic $X$ is contained in, but not necessarily equal to, the fixed subgroup $\left(\operatorname{Pic} X^{\mathrm{s}}\right)^{\Gamma_{k}}$. The Hochschild-Serre spectral sequence gives rise to an exact sequence

$$
0 \rightarrow \operatorname{Pic} X \rightarrow\left(\operatorname{Pic} X^{\mathrm{s}}\right)^{\Gamma_{k}} \rightarrow \operatorname{Br} k \rightarrow \operatorname{Br} X
$$

If $X$ has a $k$-point, then evaluation at that point gives a left inverse to $\operatorname{Br} k \rightarrow \operatorname{Br} X$, showing that $\mathrm{Pic} X \rightarrow\left(\operatorname{Pic} X^{\mathrm{s}}\right)^{\Gamma_{k}}$ is an isomorphism. In general this does not have to be true. However, because $\mathrm{Br} k$ is torsion and $\mathrm{Pic} X^{\mathrm{S}}$ is finitely generated, the above sequence shows that $\operatorname{Pic} X$ is of finite index in $\left(\operatorname{Pic} X^{\mathrm{s}}\right)^{\Gamma_{k}}$.

It is easy to see that the action of $R_{X}$ on $\mathrm{Pic} X^{\mathrm{s}}$ preserves $\left(\mathrm{Pic} X^{\mathrm{s}}\right)^{\Gamma_{k}}$, but it is not immediately obvious that this action preserves Pic $X$. To show that this is the case, we use an explicit description of $R_{X}$ provided by a theorem of Hée and Lusztig, for which Geck and Iancu gave a simple proof. Before stating their theorem, we establish some notation and conventions for Coxeter systems.

Definition 3.4 Let $W$ be a group generated by a set $T \subset W$ of elements of order 2 . For $t_{i}, t_{j} \in T$, let $n_{i, j}=n_{j, i}$ be the order of $t_{i} t_{j}$ if $t_{i} t_{j}$ has finite order and 0 otherwise. Suppose that the relations $t_{i}^{2}=1,\left(t_{i} t_{j}\right)^{n_{i, j}}=1$ for $i, j$ with $n_{i, j} \neq 0$ are a presentation of $W$. Then $(W, T)$ is a Coxeter system.

Let $G$ be a graph with vertices $T$ and such that $t_{i}, t_{j}$ are adjacent in $G$ if and only if $t_{i}$ does not commute with $t_{j}$; in this case, label the edge joining $t_{i}$ to $t_{j}$ with $n_{i, j}-2$ for $n_{i, j}>0$ and 0 otherwise. We refer to $G$ as the Coxeter-Dynkin diagram of $(W, T)$. The Coxeter system $(W, T)$ is said to be irreducible if its Coxeter-Dynkin diagram is connected.

Let the length $\ell(w)$ of an element $w \in W$ be the length of a shortest word in the $t_{i}$ that represents it. If $W$ is finite, there is $w_{0} \in W$ such that $\ell\left(w_{0}\right)>\ell(w)$ for all $w \neq w_{0} \in W$ (see [2, Proposition 2.3.1]). We refer to $w_{0}$ as the longest element of $W$.

Let $\sigma$ be a permutation of $T$. Then there is at most one way to extend $\sigma$ to a homomorphism $W \rightarrow W$, because $T$ generates $W$. If there is such an extension, it is an automorphism, because $\sigma^{-1}$ extends to its inverse, and we speak of it as the automorphism induced by $\sigma$.

If $(W, T)$ is a Coxeter system, and $I$ is a subset of $T$, let $W_{I}$ denote the subgroup of $W$ generated by the elements of $I$. Then $\left(W_{I}, I\right)$ is a Coxeter system: see [2, Proposition 2.4.1(i)]. 
Theorem 3.5 ([11, Theorem 1]) Let $(W, T)$ be a Coxeter system. Let $G$ be a group of permutations of $T$ that induce automorphisms of $W$. Let $F$ be the set of orbits $I \subset T$ for which $W_{I}$ is finite, and for $I \in F$ let $w_{I, 0}$ be the longest element of $\left(W_{I}, I\right)$. Then $\left(W^{G},\left\{w_{I, 0} \mid I \in F\right\}\right)$ is a Coxeter system.

We will apply this theorem with $W=\mathrm{W}\left(\operatorname{Pic} X^{\mathrm{s}}\right)$ and $T$ being the set of reflections in (-2)-curves on $X^{\mathrm{s}}$.

Proposition 3.6 Let $F$ be the set of Galois orbits I of (-2)-curves on $X^{\mathrm{s}}$ of the following two types:

(i) I consists of disjoint (-2)-curves;

(ii) I consists of disjoint pairs of (-2)-curves, each pair having intersection number 1.

Then the following statements hold.

(1) For each $I \in F$, let $W_{I}$ be the subgroup of $\mathrm{W}\left(\mathrm{Pic} X^{\mathrm{s}}\right)$ generated by reflections in the classes of curves in $I$, and let $r_{I}$ be the longest element of the Coxeter system $\left(W_{I}, I\right)$. Then $\left(R_{X},\left\{r_{I} \mid I \in F\right\}\right)$ is a Coxeter system.

(2) For each $I \in F$, let $C_{I} \in\left(\operatorname{Pic} X^{\mathrm{s}}\right)^{\Gamma_{k}}$ be the sum of the classes in I. Then $r_{I}$ acts on $\left(\operatorname{Pic} X^{\mathrm{s}}\right)^{\Gamma_{k}}$ as a reflection in the class $C_{I}$.

(3) The action of $R_{X}$ on $\mathrm{Pic} X^{\mathrm{s}}$ preserves $\mathrm{Pic} X$.

Proof Let $I$ be a Galois orbit of $(-2)$-curves, and suppose that the subgroup $W_{I}$ is finite. We will show that $I$ is of one of the two types described. Firstly, no two (-2)-curves in $I$ have intersection number greater than 1, for then the corresponding reflections would generate an infinite dihedral subgroup of $W_{I}$. Since $W_{I}$ is finite, its Coxeter-Dynkin diagram is a finite union of trees [2, Exercise 1.4]. In particular, it contains a vertex of degree $\leqslant 1$. However, the Galois group $\Gamma_{k}$ acts transitively on the diagram, so we conclude that either every vertex has degree 0 , or every vertex has degree 1 . These two possibilities correspond to the two types of orbits described. Now (1) follows from Theorem 3.5.

We prove (2) separately for the two types of orbits. In the first case we have $I=$ $\left\{E_{1}, \ldots, E_{r}\right\}$. The reflections in the $E_{i}$ all commute, so $W_{I}$ is isomorphic to the Coxeter group $A_{1}^{r}$. The longest element is $r_{I}=r_{E_{1}} \circ \cdots \circ r_{E_{r}}$. For $D \in\left(\operatorname{Pic} X^{\mathrm{s}}\right)^{\Gamma_{k}}$, the intersection numbers $D \cdot E_{i}$ are all equal, and one calculates

$$
r_{I}(D)=D+\left(D \cdot E_{1}\right)\left(E_{1}+\cdots+E_{r}\right)
$$

that is, $r_{I}$ coincides on $\left(\operatorname{Pic} X^{\mathrm{s}}\right)^{\Gamma_{k}}$ with reflection in the class $C_{I}=E_{1}+\cdots+E_{r}$, of self-intersection $-2 r$.

In the second case, write $I=\left\{E_{1}, E_{1}^{\prime}, \ldots, E_{r}, E_{r}^{\prime}\right\}$, where $E_{i} \cdot E_{i}^{\prime}=1$ and the other intersection numbers are all zero. The two reflections $r_{E_{i}}$ and $r_{E_{i}^{\prime}}$ generate a subgroup isomorphic to the Coxeter group $A_{2}$, in which the longest element is

$$
r_{I}=r_{E_{i}} \circ r_{E_{i}^{\prime}} \circ r_{E_{i}}=r_{E_{i}^{\prime}} \circ r_{E_{i}} \circ r_{E_{i}^{\prime}}=r_{E_{i}+E_{i}^{\prime}}
$$


Thus we have $W_{I} \cong A_{2}^{r}$ and the longest element in $W_{I}$ is the product of the longest elements in the factors $A_{2}$, that is, it equals $r_{I}=r_{E_{1}+E_{1}^{\prime}} \circ \cdots \circ r_{E_{r}+E_{r}^{\prime}}$. For $D \in$ $\left(\operatorname{Pic} X^{\mathrm{s}}\right)^{\Gamma_{k}}$, all the $D \cdot E_{i}$ and $D \cdot E_{i}^{\prime}$ are equal, and we have

$$
r_{I}(D)=D+2\left(D \cdot E_{1}\right)\left(E_{1}+E_{1}^{\prime}+\cdots+E_{r}+E_{r}^{\prime}\right)
$$

which coincides with reflection of $D$ in the class $C_{I}=E_{1}+E_{1}^{\prime}+\cdots+E_{r}+E_{r}^{\prime}$, of self-intersection $-2 r$.

Finally, each class $C_{I}$ is, by construction, the class of a Galois-fixed divisor on $X^{\mathrm{s}}$, so lies in Pic $X$. So in both cases the formula for $r_{I}$ given above shows that reflection in $C_{I}$ preserves Pic $X$, and therefore the action of $R_{X}$ preserves Pic $X$, proving (3).

We now turn to the ample and nef cones. As ampleness is a geometric property, and the nef cone is the closure of the ample cone over any base field, it follows that

$$
\operatorname{Amp}(X)=\operatorname{Amp}(\bar{X}) \cap(\operatorname{Pic} X)_{\mathbb{R}} \quad \text { and } \quad \operatorname{Nef}(X)=\operatorname{Nef}(\bar{X}) \cap(\operatorname{Pic} X)_{\mathbb{R}}
$$

the intersections taking place inside $(\operatorname{Pic} \bar{X})_{\mathbb{R}}$. The following result is well known when $k$ is algebraically closed (see [12, Corollary 8.2.11]), and descends easily to arbitrary $k$.

Proposition 3.7 Let $X$ be a K3 surface over $k$. The cone $\operatorname{Nef}(X) \cap \mathcal{C}_{X}$ is a fundamental domain for the action of $R_{X}$ on the positive cone $\mathcal{C}_{X}$, and this action is faithful.

Proof We will prove two things: first, that every class in $\mathcal{C}_{X}$ is $R_{X}$-equivalent to an element of $\operatorname{Nef}(X) \cap \mathcal{C}_{X}$; and second, that the translates of $\operatorname{Nef}(X) \cap \mathcal{C}_{X}$ by two distinct elements of $R_{X}$ meet only along their boundaries. The second of these shows in particular that the action is faithful. (When we refer to the boundary of $\operatorname{Nef}(X)$ or one of its translates, we mean the boundary within $(\operatorname{Pic} X)_{\mathbb{R}}$. The boundary of $\operatorname{Nef}(X) \cap \mathcal{C}_{X}$ in $\mathcal{C}_{X}$ is just the boundary of $\operatorname{Nef}(X)$ intersected with $\mathcal{C}_{X}$, so that distinction is not so important.)

To prove the first statement, let $D \in \mathcal{C}_{X}$. Suppose first that $D$ has trivial stabilizer in $\mathrm{W}(\operatorname{Pic} \bar{X})$. Then, by [12, Corollary 8.2.11], there exists a unique $g \in \mathrm{W}(\operatorname{Pic} \bar{X})=$ $\mathrm{W}\left(\operatorname{Pic} X^{\mathrm{s}}\right)$ such that $g D$ lies in the interior of $\operatorname{Nef}(\bar{X}) \cap \mathcal{C}_{\bar{X}}$. We claim that $g$ lies in $R_{X}$. For any $\sigma \in \Gamma_{k}$, we have

$$
\left({ }^{\sigma} g\right) D=\sigma\left(g\left(\sigma^{-1} D\right)\right)=\sigma(g(D)) \in \operatorname{Nef}(\bar{X}) \cap \mathcal{C}_{\bar{X}},
$$

since the Galois action preserves the properties of being nef and positive. By uniqueness of $g$, we conclude that $g={ }^{\sigma} g$, that is, $g$ lies in $R_{X}$. It then follows that $g D$ lies in $\left(\left(\operatorname{Pic} X^{\mathrm{s}}\right)_{\mathbb{R}}\right)^{\Gamma_{k}}=(\operatorname{Pic} X)_{\mathbb{R}}$ and therefore in $\operatorname{Nef}(X) \cap \mathcal{C}_{X}$.

Now suppose that $D \in \mathcal{C}_{X}$ has non-trivial stabilizer. Then $D$ lies on at least one of the walls defined by the action of $\mathrm{W}(\operatorname{Pic} \bar{X})$ on $(\operatorname{Pic} \bar{X})_{\mathbb{R}}$; see $[12$, Section 8.2]. By [12, Proposition 8.2.4], the chamber structure of this group action is locally polyhedral within $\mathcal{C}_{\bar{X}}$, so a small enough neighbourhood of $D$ meets only finitely many chambers. Also note that Pic $X$ is not contained in any of the walls, because $X$ admits an ample divisor. This allows us to construct a sequence $\left(D_{i}\right)_{i=1}^{\infty}$ of elements of $\mathcal{C}_{X}$, tending to $D$ 
and all lying in the interior of the same chamber of $\mathcal{C}_{\bar{X}}$. As in the previous paragraph, there is a unique $g \in R_{X}$ satisfying $g D_{i} \in \operatorname{Nef}(X) \cap \mathcal{C}_{X}$ for all $i$. By continuity, $g D$ also lies in $\operatorname{Nef}(X) \cap \mathcal{C}_{X}$.

We now prove the second statement, that $\operatorname{Nef}(X) \cap \mathcal{C}_{X}$ intersects the translate by any non-trivial element of $R_{X}$ only in its boundary. Suppose that $x \in \mathcal{C}_{X}$ lies in the intersection $\operatorname{Nef}(X) \cap g \operatorname{Nef}(X)$, for some non-trivial $g \in R_{X}$. By [12, Corollary 8.2.11], we see that $x$ lies in the boundary of $\operatorname{Nef}(\bar{X})$. The following lemma shows that $x$ lies in the boundary of $\operatorname{Nef}(X)$.

Lemma 3.8 Let $V$ be a real vector space, let $C \subset V$ be a closed convex cone, and let $S \subset V$ be a subspace having non-empty intersection with the interior of $C$. Then we have

$$
\partial_{S}(C \cap S)=\partial_{V}(C) \cap S .
$$

Proof The dual cone $C^{*} \subset V^{*}$ is defined by

$$
C^{*}=\left\{\phi \in V^{*} \mid \phi(x) \geqslant 0 \text { for all } x \in C\right\} .
$$

By the supporting hyperplane theorem, $C^{*}$ has the property that a point $x \in C$ lies in $\partial_{V}(C)$ if and only if there exists a non-zero $\phi \in C^{*}$ satisfying $\phi(x)=0$. (The hyperplane $\phi=0$ is called a supporting hyperplane of $C$ at $x$.) Let $f: V^{*} \rightarrow S^{*}$ be the natural restriction map; then the dual $(C \cap S)^{*}$ is equal to $f\left(C^{*}\right)$ (see [16, Corollary 16.3.2]).

Let $x$ be a point of $\partial_{V}(C) \cap S$. Then there is a supporting hyperplane to $C$ at $x$, that is, there exists a non-zero $\phi \in C^{*}$ satisfying $\phi(x)=0$. The condition that $S$ meet the interior of $C$ implies that $\phi$ does not vanish identically on $S$, so $f(\phi)$ is non-zero. Thus $f(\phi)$ is a non-zero element of $(C \cap S)^{*}$ vanishing at $x$, so $x$ lies in $\partial_{S}(C \cap S)$.

Conversely, suppose that $x$ lies in $\partial_{S}(C \cap S)$. Then there is a supporting hyperplane to $C \cap S$ at $x$, that is, there exists a non-zero $\psi \in(C \cap S)^{*}$ satisfying $\psi(x)=0$. Let $\phi \in C^{*}$ satisfy $f(\phi)=\psi$; then we have $\phi(x)=0$ and so $x \in \partial_{V}(C)$.

Remark 3.9 We can also give an explicit description of the walls of $\operatorname{Nef}(X) \cap \mathcal{C}_{X}$. According to [12, Corollary 8.1.6], a class in $\mathcal{C}_{X}$ lies in $\operatorname{Nef}(X)$ if and only if it has non-negative intersection number with every $(-2)$-curve on $X^{\mathrm{s}}$, or, equivalently, with every Galois orbit of $(-2)$-curves. The question is to determine which Galois orbits are superfluous, and which actually define walls of $\operatorname{Nef}(X) \cap \mathcal{C}_{X}$.

Let $I$ be a Galois orbit of $(-2)$-curves on $X^{\mathrm{s}}$, and suppose that the subgroup $W_{I} \subset$ $\mathrm{W}\left(\mathrm{Pic} X^{\mathrm{s}}\right)$ generated by reflections in the elements of $I$ is finite, that is, $I$ is as described in Proposition 3.6. The longest element of $W_{I}$ acts on Pic $X$ by reflection in the class $C_{I}=\sum_{E \in I} E$, which has negative self-intersection. The hyperplane orthogonal to $C_{I}$ is a wall of $\operatorname{Nef}(X) \cap \mathcal{C}_{X}$ : by the same argument as in [20], given a class $D \in \operatorname{Amp}(X)$, the class

$$
D-\frac{D \cdot C_{I}}{C_{I} \cdot C_{I}} C_{I}
$$

is orthogonal to $C_{I}$ but has positive intersection number with all (-2)-curves outside $I$. 
On the other hand, let $I$ be a Galois orbit of $(-2)$-curves such that the subgroup $W_{I}$ is infinite. Then, for a (-2)-curve $C \in I$, the hyperplane orthogonal to $C$ in $\left(\operatorname{Pic} X^{\mathrm{s}}\right)_{\mathbb{R}}$ does not meet $\operatorname{Nef}(X) \cap \mathcal{C}_{X}$, as the following argument shows. Let $x$ be a class in $\mathcal{C}_{X}$ orthogonal to $C$; then $x$ is also orthogonal to all the other curves in $I$, and so is fixed by $W_{I}$. Therefore $x$ is also orthogonal to the infinitely many images of $C$ under the action of $W_{I}$, contradicting the fact that the chamber structure induced by $\mathrm{W}\left(\operatorname{Pic} X^{\mathrm{s}}\right)$ on $\mathcal{C}_{X^{\mathrm{s}}}$ is locally polyhedral.

Next we would like to prove an analogue of Theorem 1.1(2). However, while the authors do not have an explicit counterexample, there seems to be no reason for the image of $R_{X}$ in $\mathrm{O}$ (Pic $X$ ) to be normal. Instead, we will see that there is a natural homomorphism from a semidirect product Aut $X \ltimes R_{X}$ to $\mathrm{O}$ (Pic $X$ ) having finite kernel and image of finite index.

Note that the natural action of Aut $X \subset$ Aut $\bar{X}$ on $\mathrm{O}(\operatorname{Pic} \bar{X})$ by conjugation fixes $\mathrm{W}(\operatorname{Pic} \bar{X})$ and commutes with the Galois action, so fixes $R_{X}$. This gives an action of Aut $X$ on $R_{X}$, and a homomorphism from the associated semidirect product Aut $X \ltimes R_{X}$ to $\mathrm{O}$ (Pic $\bar{X}$ ). Since Aut $X$ and $R_{X}$ both fix Pic $X$, we also obtain a homomorphism Aut $X \ltimes R_{X} \rightarrow \mathrm{O}(\operatorname{Pic} X)$.

Proposition 3.10 Let X be a K3 surface over a field $k$ of characteristic different from 2. Then the natural map

$$
\text { Aut } X \ltimes R_{X} \rightarrow \mathrm{O}(\operatorname{Pic} X)
$$

\section{has finite kernel and image of finite index.}

Remark 3.11 In the literature, this statement appears in various different but equivalent forms.

- Because the action of Aut $X$ fixes the ample cone, the image of Aut $X$ in $\mathrm{O}(\mathrm{Pic} X)$ meets $R_{X}$ only in the identity element. Therefore the finiteness of the kernel in Proposition 3.10 is equivalent to the finiteness of the kernel of Aut $X \rightarrow \mathrm{O}(\operatorname{Pic} X)$.

- Let Aut $_{\mathrm{s}} X \subset$ Aut $X$ be the subgroup of symplectic automorphisms [12, Definition 15.1.1]. Over an algebraically closed field $k$ of characteristic zero, the induced map from $\operatorname{Aut}_{\mathrm{s}} X$ to $\mathrm{O}(\mathrm{Pic} X)$ is injective, so that $\operatorname{Aut}_{\mathrm{s}} X \ltimes \mathrm{W}$ (Pic $X$ ) can be viewed as a subgroup of $\mathrm{O}(\operatorname{Pic} X)$. Instead of our Proposition 3.10, Huybrechts [12, Theorem 15.2.6] makes the statement that Aut $_{\mathrm{s}} X \ltimes \mathrm{W}(\operatorname{Pic} X)$ has finite index in $\mathrm{O}(\operatorname{Pic} X)$. This is equivalent to our formulation, because $\mathrm{Aut}_{\mathrm{s}} X$ is of finite index in Aut $X$. However, when $k$ is not algebraically closed there is no reason for Aut $_{\mathrm{s}} X \rightarrow \mathrm{O}(\mathrm{Pic} X)$ to be injective, so there is no advantage to stating the result in terms of $\mathrm{Aut}_{\mathrm{s}} X$.

- Lieblich and Maulik [13, Theorem 6.1] define $\Gamma \subset \mathrm{O}(\operatorname{Pic} X)$ to be the subgroup of elements preserving the ample cone, and then show that Aut $X \rightarrow \Gamma$ has finite kernel and cokernel. Over an algebraically closed base field $k$, the subgroups $\Gamma$ and $\mathrm{W}(\mathrm{Pic} X)$ generate $\mathrm{O}(\mathrm{Pic} X)$ : any element of $\mathrm{O}(\mathrm{Pic} X)$ permutes the $(-2)$-classes in Pic $X$ and therefore takes the ample cone to one of its translates under W(Pic $X$ ); so composing with an element of $\mathrm{W}(\operatorname{Pic} X)$ gives an element of $\Gamma$. Therefore that condition is also equivalent to the condition of Proposition 3.10. 
Before proving Proposition 3.10, we first state two lemmas which are well known in the case of abelian groups.

Lemma 3.12 Let $G$ be a finite group, and $f: A \rightarrow B$ a homomorphism of (possibly non-commutative) $G$-modules having finite kernel and image of finite index. Then the induced homomorphism $f^{G}: A^{G} \rightarrow B^{G}$ also has finite kernel and image of finite index.

Proof The kernel of $f^{G}$ is contained in the kernel of $f$, so is finite. For the statement about the image, consider the short exact sequence

$$
0 \rightarrow \operatorname{ker} f \rightarrow A \stackrel{f}{\rightarrow} \operatorname{im} f \rightarrow 0 .
$$

The associated exact sequence of cohomology gives

$$
0 \rightarrow(\operatorname{ker} f)^{G} \rightarrow A^{G} \stackrel{f^{G}}{\longrightarrow}(\operatorname{im} f)^{G} \rightarrow \mathrm{H}^{1}(G, \operatorname{ker} f)
$$

and $\mathrm{H}^{1}(G, \operatorname{ker} f)$ is a finite set, showing that $\operatorname{im}\left(f^{G}\right)$ is of finite index in $(\operatorname{im} f)^{G}$. On the other hand, we claim that $(\operatorname{im} f)^{G}$ is of finite index in $B^{G}$. Indeed, by hypothesis $\operatorname{im}(f)$ is of finite index in $B$, and this property is preserved on intersecting with the subgroup $B^{G}$. Thus $\operatorname{im}\left(f^{G}\right)$ is of finite index in $B^{G}$.

The following lemma is standard and easy to prove; we state it for reference.

Lemma 3.13 Let $f: A \rightarrow B$ and $g: B \rightarrow C$ be two homomorphisms of groups.

(1) If $f$ and $g$ both have finite kernel and image of finite index, then so does the composition $g \circ f$.

(2) If $g \circ f$ has finite kernel and image of finite index, and $g$ has finite kernel, then $f$ has finite kernel and image of finite index.

(3) If $g \circ f$ has finite kernel and image of finite index, and $f$ has image of finite index, then $g$ has finite kernel and image of finite index.

Proof We leave this as an exercise for the reader.

Proof of Proposition 3.10 By [12, Theorem 15.2.6] in characteristic zero, or [13] in characteristic $p>2$, the natural map

$$
\text { Aut } \bar{X} \rightarrow \mathrm{O}(\operatorname{Pic} \bar{X}) / \mathrm{W}(\operatorname{Pic} \bar{X})
$$

has finite kernel and image of finite index. Lemma 3.1 shows that the same is true for Aut $X^{\mathrm{s}} \rightarrow \mathrm{O}\left(\mathrm{Pic} X^{\mathrm{s}}\right) / \mathrm{W}\left(\mathrm{Pic} X^{\mathrm{s}}\right)$. The action of $\Gamma_{k}$ on all of these groups factors through a finite quotient, so Lemma 3.12 shows that the induced homomorphism

$$
\left(\text { Aut } X^{\mathrm{s}}\right)^{\Gamma_{k}} \rightarrow\left(\mathrm{O}\left(\operatorname{Pic} X^{\mathrm{s}}\right) / \mathrm{W}\left(\operatorname{Pic} X^{\mathrm{s}}\right)\right)^{\Gamma_{k}}
$$


also has finite kernel and image of finite index. Because the automorphism group functor is representable, we have (Aut $\left.X^{\mathrm{s}}\right)^{\Gamma_{k}}=$ Aut $X$.

There is an exact sequence

$$
1 \rightarrow R_{X} \rightarrow \mathrm{O}\left(\operatorname{Pic} X^{\mathrm{s}}\right)^{\Gamma_{k}} \rightarrow\left(\mathrm{O}\left(\operatorname{Pic} X^{\mathrm{s}}\right) / \mathrm{W}\left(\operatorname{Pic} X^{\mathrm{s}}\right)\right)^{\Gamma_{k}}
$$

and the homomorphism (3.1) factors through $\mathrm{O}\left(\operatorname{Pic} X^{\mathrm{s}}\right)^{\Gamma_{k}}$, and hence through the injective map

$$
\mathrm{O}\left(\operatorname{Pic} X^{\mathrm{s}}\right)^{\Gamma_{k}} / R_{X} \rightarrow\left(\mathrm{O}\left(\operatorname{Pic} X^{\mathrm{s}}\right) / \mathrm{W}\left(\operatorname{Pic} X^{\mathrm{s}}\right)\right)^{\Gamma_{k}}
$$

Therefore, by Lemma 3.13, the map

$$
\text { Aut } X \rightarrow \mathrm{O}\left(\operatorname{Pic} X^{\mathrm{s}}\right)^{\Gamma_{k}} / R_{X}
$$

has finite kernel and image of finite index. Since the image of Aut $X$ in $\mathrm{O}\left(\operatorname{Pic} X^{s}\right)^{\Gamma_{k}}$ meets $R_{X}$ only in the identity element, this shows that the natural map

$$
\text { Aut } X \ltimes R_{X} \rightarrow \mathrm{O}\left(\operatorname{Pic} X^{s}\right)^{\Gamma_{k}}
$$

also has finite kernel and image of finite index.

We apply Proposition 2.2 with $\Lambda=\operatorname{Pic} X^{\mathrm{s}}$ and $H$ being the image of $\Gamma_{k}$ in $\mathrm{O}\left(\mathrm{Pic} X^{\mathrm{s}}\right)$. The centralizer $Z_{\mathrm{O}(\Lambda)}(H)$ is $\mathrm{O}\left(\operatorname{Pic} X^{\mathrm{s}}\right)^{\Gamma_{k}}$, so part (2) of Proposition 2.2 shows that $\mathrm{O}\left(\operatorname{Pic} X^{\mathrm{s}}\right)^{\Gamma_{k}}$ is of finite index in $\mathrm{O}\left(\operatorname{Pic} X^{\mathrm{s}}\right.$, $\left.\left(\operatorname{Pic} X^{\mathrm{s}}\right)^{\Gamma_{k}}\right)$. Parts (1) and (2) of Proposition 2.2 combined show that the map

$$
\mathrm{O}\left(\operatorname{Pic} X^{\mathrm{s}},\left(\operatorname{Pic} X^{\mathrm{s}}\right)^{\Gamma_{k}}\right) \rightarrow \mathrm{O}\left(\left(\operatorname{Pic} X^{\mathrm{s}}\right)^{\Gamma_{k}}\right)
$$

has finite kernel and image of finite index; by Lemma 3.13 so does the map $\mathrm{O}\left(\operatorname{Pic} X^{\mathrm{s}}\right)^{\Gamma_{k}} \rightarrow \mathrm{O}\left(\left(\operatorname{Pic} X^{\mathrm{s}}\right)^{\Gamma_{k}}\right)$.

Composing with (3.2) and applying Lemma 3.13 shows that

$$
\text { Aut } X \ltimes R_{X} \rightarrow \mathrm{O}\left(\left(\operatorname{Pic} X^{\mathrm{s}}\right)^{\Gamma_{k}}\right)
$$

has finite kernel and image of finite index. Since the actions of both Aut $X$ and $R_{X}$ on $\left(\operatorname{Pic} X^{\mathrm{s}}\right)^{\Gamma_{k}}$ preserve Pic $X$, this last map factors as

$$
\text { Aut } X \ltimes R_{X} \rightarrow \mathrm{O}\left(\left(\operatorname{Pic} X^{\mathrm{s}}\right)^{\Gamma_{k}}, \operatorname{Pic} X\right) \rightarrow \mathrm{O}\left(\left(\operatorname{Pic} X^{\mathrm{s}}\right)^{\Gamma_{k}}\right) \text {. }
$$

As the second map in this composition is clearly injective, Lemma 3.13 shows that the first one has finite kernel and image of finite index.

Finally, Pic $X$ is of finite index in $\left(\operatorname{Pic} X^{\mathrm{s}}\right)^{\Gamma_{k}}$, so its orthogonal complement in the latter is trivial. Lemma 2.3(2) shows that the natural map

$$
\mathrm{O}\left(\left(\operatorname{Pic} X^{\mathrm{s}}\right)^{\Gamma_{k}}, \operatorname{Pic} X\right) \rightarrow \mathrm{O}(\operatorname{Pic} X)
$$


is injective, and its image has finite index. Composing with the first map of (3.3) and applying Lemma 3.13 again, we deduce that Aut $X \ltimes R_{X} \rightarrow \mathrm{O}(\operatorname{Pic} X)$ has finite kernel and image of finite index.

Remark 3.14 The finiteness of the kernel can also be proved directly, by the same proof as over an algebraically closed field.

Having proved Proposition 3.10, we can deduce the remaining results exactly as in the classical case. Define the cone $\operatorname{Nef}^{\mathrm{e}}(X)$ to be the real convex hull of $\operatorname{Nef}(X) \cap \operatorname{Pic} X$.

Corollary 3.15 The action of Aut $X$ on $\mathrm{Nef}^{\mathrm{e}}(X)$ admits a rational polyhedral fundamental domain.

Proof This is as in the case of $k=\mathbb{C}$; we briefly recall the argument of Sterk [20], making the necessary adjustments.

Let $\Lambda$ be a lattice of signature $(1, \rho-1)$ and let $\Gamma \subset \mathrm{O}\left(\Lambda_{\mathbb{R}}\right)$ be an arithmetic subgroup (for example, a subgroup of finite index in $\mathrm{O}(\Lambda)$ ). Let $C \subset \Lambda_{\mathbb{R}}$ be one of the two components of $\left\{x \in \Lambda_{\mathbb{R}} \mid(x \cdot x)<0\right\}$; this is a self-adjoint homogeneous cone $\left[1\right.$, Remark 1.11]. Let $C_{+}$be the convex hull of $\bar{C} \cap \Lambda_{\mathbb{Q}}$. Pick any $y \in C \cap \Lambda$; the argument of $[20, \mathrm{p} .511]$ shows that the set

$$
\Pi=\left\{x \in C_{+} \mid(\gamma x \cdot y) \geqslant(x \cdot y) \text { for all } \gamma \in \Gamma\right\}
$$

is rational polyhedral (for this, the reference to Proposition 11 of the first edition of [1] has become Proposition 5.22 in the second edition) and is a fundamental domain for the action of $\Gamma$ on $C_{+}$. (Sterk does not explicitly prove that two translates of $\Pi$ intersect only in their boundaries, but this is easy to show from the description above.) In our case, applying this with $\Lambda=\operatorname{Pic} X$ and $\Gamma$ being the image of Aut $X \ltimes R_{X} \rightarrow \mathrm{O}(\operatorname{Pic} X)$ gives a rational polyhedral fundamental domain $\Pi$ for the action of Aut $X \ltimes R_{X}$ on $\left(\mathrm{C}_{X}\right)_{+}$.

If we choose $y$ to be an ample class in Pic $X$, then the resulting $\Pi$ is contained in $\operatorname{Nef}(X)$, as we now show. Let $I$ be a Galois orbit of $(-2)$-curves on $X^{\mathrm{s}}$ such that the corresponding group $W_{I} \subset \mathrm{W}\left(\operatorname{Pic} X^{\mathrm{s}}\right)$ is finite. Proposition 3.6 states that the longest element $w$ of $W_{I}$ acts on Pic $X$ as reflection in the class $C_{I}=\sum_{E \in I} E$, and that these elements generate $R_{X}$. Taking $\gamma=w$ in the definition of $\Pi$ shows that $\Pi$ is contained in the half-space $\left\{x \mid x . C_{I} \geqslant 0\right\}$. As this holds for all such $I$, Remark 3.9 shows that $\Pi$ is contained in $\operatorname{Nef}(X)$.

We conclude as in [20]. If $x$ is a class in $\operatorname{Nef}^{\mathrm{e}}(X)$ then, since $\Pi$ is a fundamental domain for the action of Aut $X \ltimes R_{X}$ on $\left(\bigodot_{X}\right)_{+}$, we can find $\phi \in$ Aut $X$ and $r \in R_{X}$ such that $r \phi(x)$ lies in $\Pi$. But now $\phi(x)$ and $r \phi(x)$ both lie in $\operatorname{Nef}^{\mathrm{e}}(X)$, so they are equal and lie in $\Pi$. This shows that $\Pi$ is a fundamental domain for the action of Aut $X$ on $\operatorname{Nef}^{\mathrm{e}}(X)$.

Corollary 3.16 (1) There are only finitely many Aut X-orbits of k-rational (-2)curves on $X$.

(2) There are only finitely many Aut $X$-orbits of primitive Picard classes of irreducible curves on $X$ of arithmetic genus 1. 
(3) For $g \geqslant 2$, there are only finitely many Aut X-orbits of Picard classes of irreducible curves on $X$ of arithmetic genus $g$.

Proof Let $\Pi$ be a rational polyhedral fundamental domain for the action of Aut $X$ on $\mathrm{Nef}^{\mathrm{e}}(X)$, as in Corollary 3.15. Every $k$-rational (-2)-curve on $X$ defines a wall of $\operatorname{Nef}(X)$, by Remark 3.9. Since $\Pi$ meets only finitely many walls of $\operatorname{Nef}(X)$, this proves (1).

Gordan's Lemma states that the integral points of the dual cone of a rational polyhedral convex cone form a finitely generated monoid. Applying this to the dual cone of $\Pi$, let $D_{1}, \ldots, D_{r}$ be a minimal set of generators for $\Pi \cap P i c X$. Since these all lie in $\operatorname{Nef}(X)$, we have $D_{i} \cdot D_{i} \geqslant 0$ for all $i$, and $D_{i} \cdot D_{j}>0$ for $i \neq j$. As observed in [20], this implies that, for any $n>0$, there are only finitely many classes in $\Pi \cap \operatorname{Pic} X$ of self-intersection $n$; and there are only finitely many primitive classes in $\Pi \cap \operatorname{Pic} X$ of self-intersection zero. The class of an irreducible curve of arithmetic genus $g \geqslant 1$ has self-intersection $2 g-2$ and therefore lies in $\operatorname{Nef}(X)$, so this proves (2) and (3).

Remark 3.17 It is not true that every irreducible curve on $X$ of arithmetic genus 0 is a $k$-rational (-2)-curve. However, there are not many possibilities, as we now show. Let $C$ be such a curve, and let $C_{1}, \ldots, C_{r}$ be the geometric components of $C$; the Galois group $\Gamma_{k}$ acts transitively on them. In order to achieve $C^{2}=-2$, we must have $C_{i}^{2}<0$ for all $i$, so each $C_{i}$ is a $(-2)$-curve. Consider the intersection matrix $\left(C_{i} \cdot C_{j}\right)$ : the sum of the entries in the matrix is $C^{2}=-2$, and the Galois action shows that every row sum is the same. Therefore the number $r$ of rows divides 2 , and there are only two options: $r=1$, so that $C$ is a rational (-2)-curve; or $r=2$, and $C=C_{1} \cup C_{2}$ is the union of two conjugate $(-2)$-curves meeting transversely in a single point. By Corollary 3.15 , both types of curves define walls of $\operatorname{Nef}(X)$, so in fact both types fall into finitely many orbits under the action of Aut $(X)$.

Both types occur on the surfaces considered in Sect. 4.3. Indeed, the line $x=y$, $z=w$ on the surface $x^{4}-y^{4}=c\left(z^{4}-w^{4}\right)$ is a rational (-2)-curve, while the line $x=y, z=i w$ meets its conjugate transversely in a single point.

Remark 3.18 In the case $g=1$, the condition that the class be primitive cannot be omitted, for the following reason. Take for example $k=\mathbb{Q}$, and suppose that $X$ admits an elliptic fibration $\pi: X \rightarrow \mathbb{P}^{1}$. A general fibre of such a fibration is a smooth, geometrically irreducible curve $E$ of genus 1, whose class in Pic $X$ has self-intersection 0 and is primitive. (In fact, the class is even primitive in Pic $\bar{X}$.) If $s \in \mathbb{P}^{1}$ is a point of degree $m>1$, then in general the fibre $\pi^{-1}(s)$ will be an irreducible curve on $X$ of arithmetic genus 1 , linearly equivalent to $m E$. As $m$ varies, this construction gives infinitely many such classes that are clearly not Aut $X$-equivalent.

\section{Examples}

In this section we give three examples that illustrate some of the theory developed up to this point. First, we will give a K3 surface $X$ with finite automorphism group, even though all K3 surfaces $V$ over $\overline{\mathbb{Q}}$ with Pic $V \cong$ Pic $X$ have infinite automorphism group. Second, we will construct a surface $Y$ with finite automorphism group, even 
though, for all extensions $k / \mathbb{Q}$, all $\mathrm{K} 3$ surfaces $V$ over $\overline{\mathbb{Q}}$ with Pic $V \cong$ Pic $Y_{k}$ have infinite automorphism group. For a third example, we will prove that the quartic surface $Z$ in $\mathbb{P}^{3}$ defined by $x^{4}-y^{4}=c\left(z^{4}-w^{4}\right)$ over a field $k$ of characteristic 0 has finite automorphism group when $c \in k$ is such that the Galois group of the field of definition of the Picard group has degree 16, the largest possible.

\subsection{First example}

We construct a surface $X$ such that Aut $\bar{X}$ is finite, and thus so is Aut $X$, while any K3 surface over $\overline{\mathbb{Q}}$ having the same Picard lattice as $X$ has infinite automorphism group. In contrast, over an algebraically closed field of characteristic not equal to 2 , finiteness of the automorphism group depends only on the isomorphism type of the Picard lattice. This is an immediate consequence of statement (2) of Theorem 1.1.

Let $M$ and $N$ be the block diagonal matrices

$$
M=\left(\begin{array}{ccc}
0 & 1 & 0 \\
1 & 0 & 0 \\
0 & 0 & -8
\end{array}\right), \quad N=\left(\begin{array}{cc|c}
0 & 1 & \\
1 & 0 & \\
\hline & & -2 I_{4}
\end{array}\right)
$$

where $I_{4}$ is the $4 \times 4$ identity matrix. Let $L_{N}$ be a lattice with basis $\left(e_{1}, \ldots, e_{6}\right)$ and Gram matrix $N$ with respect to that basis. Let $L_{M} \subset L_{N}$ be the sublattice generated by $e_{1}, e_{2}$ and $e_{3}+e_{4}+e_{5}+e_{6}$. The Gram matrix for $L_{M}$ with respect to this basis is $M$. The surface $X$ that we will construct will have compatible isomorphisms $\operatorname{Pic} \bar{X} \cong L_{N}$ and Pic $X \cong L_{M}$.

Proposition 4.1 Let $V$ be a $K 3$ surface over $\overline{\mathbb{Q}}$ with Pic $V \cong L_{M}$. Then Aut $V$ is infinite.

Proof There is an obvious embedding of the hyperbolic lattice $U$ with Gram matrix $\left(\begin{array}{ll}0 & 1 \\ 1 & 0\end{array}\right)$ into $L_{M} \cong$ Pic $V$. By [12, Remark 11.1.4], this implies that there is an elliptic fibration $\pi: V \rightarrow \mathbb{P}^{1}$ with a section. Let $E$ be the class of a fibre of $\pi$ and $O$ the class of a section; then $E$ and $O$ generate a sublattice of Pic $V$ isomorphic to $U$ (though not necessarily the obvious one). If $\pi$ were to have a reducible fibre, then any component of that fibre other than the one meeting $O$ would lie in $\langle E, O\rangle^{\perp}$ and have self-intersection -2 . However, $\langle E, O\rangle$ is a lattice of rank 2 and determinant -1 , so the determinant of $\langle E, O\rangle^{\perp}$ is the negative of that of Pic $V$ and its rank is 2 less. Thus it is generated by a single vector of norm -8 and so there are no reducible fibres. It follows by the Shioda-Tate formula [12, Corollary 11.3.4] that the Mordell-Weil group of the fibration has rank 1. Translation by a non-torsion section gives an automorphism of $V$ of infinite order.

Remark 4.2 In fact, Shimada [18, Remark 9.3] has proved that this translation, and negation in the Mordell-Weil group, generate the whole of Aut $V$.

In contrast with Proposition 4.1, we will see that there exist K3 surfaces over $\mathbb{Q}$ with Picard lattice isomorphic to $L_{M}$ for which the automorphism group is finite. We use the standard Kodaira symbols for reducible fibres of elliptic fibrations, as in [19, IV.9, Table 4.1]. 
Proposition 4.3 Let $V$ be a K3 surface over an algebraically closed field with an elliptic fibration $\pi: V \rightarrow \mathbb{P}^{1}$ that has a section. Suppose that $\pi$ has four fibres each of type either $I_{2}$ or III. Then the following statements are equivalent.

(1) The rank of Pic $V$ is at most 6.

(2) The Picard lattice Pic $V$ is isomorphic to $L_{N}$.

(3) The Mordell-Weil group associated to $\pi$ is trivial, and there are only four reducible fibres.

If these equivalent conditions hold, then Aut $V$ is finite.

Before proving the proposition, we state and prove a lemma.

Lemma 4.4 Let $V$ be a $K 3$ surface containing four pairwise disjoint smooth rational curves $C_{1}, C_{2}, C_{3}, C_{4}$. Then the class $\sum_{i=1}^{4}\left[C_{i}\right]$ cannot be divided by 2 in Pic $V$.

Proof Suppose otherwise, and let $D=\left(\sum_{i=1}^{4}\left[C_{i}\right]\right) / 2 \in$ Pic $V$. Then $D^{2}=-2$, so either $D$ or $-D$ is effective; since $2 D$ is effective, we deduce that $D$ is effective. Now $\left(D,\left[C_{i}\right]\right)=-1$ for $1 \leqslant i \leqslant 4$, so the $C_{i}$ are base components of the linear system $|D|$ and $D-\sum_{i=1}^{4}\left[C_{i}\right]=-D$ is also effective, giving a contradiction.

Proof of Proposition 4.3 The equivalence of (2) and (3) is proved as follows. By [12, Proposition 11.3.2], there is an exact sequence

$$
0 \rightarrow A \rightarrow \operatorname{Pic} V \rightarrow G \rightarrow 0
$$

where $A \subset$ Pic $V$ is the subgroup generated by vertical divisors and a chosen section, and $G$ is the Mordell-Weil group of the elliptic fibration. Let $E$ be a fibre of $\pi$, let $O$ be a chosen section, and let $W_{1}, \ldots, W_{4}$ be the components of the four given fibres that do not meet $O$. The classes $[E],[E]+[O],\left[W_{1}\right], \ldots,\left[W_{4}\right]$ lie in $A$ and have intersection matrix equal to $N$, so they generate a sublattice of $A$ isomorphic to $L_{N}$. If $G$ is trivial and there are no other reducible fibres, then these six classes generate Pic $V$ and so we have Pic $V \cong L_{N}$. Conversely, if Pic $V$ is isomorphic to $L_{N}$ then these six classes must generate Pic $V$, so $G$ is trivial. Also, there can be no further reducible fibres, for the class of a curve in such a fibre would be independent of the given generators of Pic $V$.

The implication $(2) \Rightarrow(1)$ is trivial; we now prove $(1) \Rightarrow(2)$. As above, we have an embedding $L_{N} \hookrightarrow$ Pic $V$, so Pic $V$ must have rank exactly 6. Since Pic $V$ has rank 6 this embedding has finite index, and we must prove it to be an isomorphism. The determinant of $N$ is -16 ; the square of the index [Pic $V: L_{N}$ ] must divide this, so the index is 1,2 , or 4 . If it is not 1 , there is some element of $L_{N}$ that can be divided by 2 in Pic $V$ but not in $L_{N}$. We take this element to be of the form $a[E]+b[O]+\sum_{i=1}^{4} c_{i}\left[W_{i}\right]$, where all the coefficients are 0 or 1 and not all are 0 . Then $a=b=0$, for otherwise the intersection number with $[O]$ or $[E]$ would be odd; and all the $c_{i}$ must be equal, because the self-intersection of any divisor on a K3 surface is even and hence that of any divisor that can be divided by 2 is a multiple of 8 . Thus all $c_{i}$ are equal to 1 . However, Lemma 4.4 shows that $\sum_{i=1}^{4}\left[W_{i}\right]$ is not divisible by 2 . This proves Pic $V \cong L_{N}$.

Finally, Nikulin [14, Theorem 3.1] has listed the finitely many possibilities for Pic $V$ of rank $\geqslant 6$ that give rise to finite automorphism groups. The lattice $L_{N} \cong U \oplus 4 A_{1}$ is in the list, showing that Aut $V$ is finite. 
Proposition 4.5 Let $X$ be a K3 surface over a field $k$ with an elliptic fibration $\pi: X \rightarrow$ $\mathbb{P}^{1}$ that has a section. Suppose that $\pi$ has four conjugate fibres of type $I_{2}$ or III and that the rank of $\operatorname{Pic} \bar{X}$ is at most 6 . Then there are compatible isomorphisms $\operatorname{Pic} \bar{X} \cong L_{N}$ and $\operatorname{Pic} X \cong L_{M}$, and Aut $X$ is finite.

Proof Applying Proposition 4.3 to the surface $\bar{X}$ shows that Pic $\bar{X}$ is isomorphic to $L_{N}$. More precisely, the proof shows that there is an isomorphism $\operatorname{Pic} \bar{X} \cong L_{N}$ that identifies the basis $\left(e_{1}, \ldots, e_{6}\right)$ of $L_{N}$ with the basis $\left([E],[E]+[O],\left[W_{1}\right], \ldots,\left[W_{4}\right]\right)$ of Pic $\bar{X}$, where $E$ is a fibre of $\pi$ and $O$ is a section, and $W_{1}, \ldots, W_{4}$ are the components of the four reducible fibres that do not meet $O$. Since by Proposition 4.3 there are no other reducible fibres, the Galois action permutes $W_{1}, \ldots, W_{4}$ transitively and so the Galois-invariant subgroup is identified with $L_{M}$. As $X$ contains a $k$-rational curve $O$ of genus 0 , it has rational points over $k$ and hence $\operatorname{Pic} X=(\operatorname{Pic} \bar{X})^{\Gamma \mathbb{Q}}=L_{M}$.

Proposition 4.3 also shows that Aut $\bar{X}$ is finite, and a fortiori that Aut $X$ is finite.

We now construct a K3 surface $X$ over $\mathbb{Q}$ satisfying the conditions of Proposition 4.5, as the Jacobian of a genus-1 fibration on a quartic $U$ in $\mathbb{P}^{3}$. See [12, Sect. 11.4] for the properties of the Jacobian of a genus-1 fibration on a K3 surface.

Let $U$ be a smooth quartic surface in $\mathbb{P}^{3}$ over $\mathbb{Q}$ containing a line $L$. Projection away from $L$ induces a morphism $\pi_{L}: U \rightarrow \mathbb{P}^{1}$ whose fibres are the residual intersections with $U$ of planes containing $L$. The generic fibre is a smooth curve of genus 1, and the induced morphism $L \rightarrow \mathbb{P}^{1}$ has degree 3 .

Proposition 4.6 Let $U$ be a smooth quartic surface in $\mathbb{P}_{\mathbb{Q}}^{3}$ containing a rational line $L$ and $a$ Galois orbit $\mathcal{L}$ of four lines that meet $L$. Suppose in addition that each of the four planes containing $L$ and a line in $\mathcal{L}$ meets $U$ in one further component, which is a smooth conic. Then $\pi_{L}$ has four conjugate fibres of type $I_{2}$ or III.

Let $X \rightarrow \mathbb{P}^{1}$ be the relative Jacobian of $\pi_{L}$, and suppose in addition that $\mathrm{Pic} \bar{U}$ has rank at most 6 . Then there are compatible isomorphisms Pic $\bar{X} \cong L_{N}$ and $\operatorname{Pic} X \cong L_{M}$, and Aut $X$ is finite.

Proof Let $H$ be one of the four conjugate planes containing $L$ and a line $L^{\prime} \in \mathcal{L}$, and let $C$ denote the residual smooth conic. The union $L^{\prime} \cup C$ is a fibre of $\pi_{L}$. We have $L^{\prime} \cdot C=2$, because $L^{\prime}, C$ are a line and a conic in the plane $H$. Either $L^{\prime}$ is tangent to $C$, in which case we have a fibre of type $I I I$, or they intersect in two distinct points, and the fibre is of type $I_{2}$. The same description holds for the other three planes that are Galois conjugates of $H$.

The relative Jacobian $X$ is a $\mathrm{K} 3$ surface [12, Proposition 11.4.5] that has the same geometric Picard number as $U$ [12, Corollary 11.4.7 and the discussion following it], and $X \rightarrow \mathbb{P}^{1}$ has the same geometric fibres as $\pi_{L}$ [12, Chapter 11, Equation (4.1)]. Now apply Proposition 4.5.

A very general surface $U$ constructed according to this proposition has Picard group generated by the classes of $L$, the lines in $\mathcal{L}$ and a fibre of $\pi_{L}$. This does not imply that such a surface exists over $\mathbb{Q}$, but we will see that it is not difficult to find an example. 
Table 1 Point counts on the surface $U_{3}$

\begin{tabular}{lllllllll}
\hline$n$ & 1 & 2 & 3 & 4 & 5 & 6 & 7 & 8 \\
\hline$\# U_{3}\left(\mathbb{F}_{3^{n}}\right)$ & 16 & 94 & 676 & 7066 & 60,076 & 533,818 & $4,785,076$ & $43,101,802$ \\
\hline
\end{tabular}

Example 4.7 We claim that the surface $U \subset \mathbb{P}_{\mathbb{Q}}^{3}$ given by the equation

$$
\begin{aligned}
-2 x^{3} z & -3 x^{2} y z-3 y^{3} z+x^{2} z^{2}-3 x y z^{2}+2 y^{2} z^{2}+x z^{3}+y z^{3} \\
& -13 x^{3} w+24 x^{2} y w-13 x y^{2} w+8 y^{3} w-x^{2} z w+51 x z^{2} w \\
& -37 x^{2} w^{2}+47 x y w^{2}-16 y^{2} w^{2}+111 x z w^{2}-38 y z w^{2} \\
& -57 z^{2} w^{2}-227 x w^{3}+24 y w^{3}-94 z w^{3}+303 w^{4}=0
\end{aligned}
$$

satisfies the conditions of Proposition 4.6. Indeed, $U$ contains the line $L$ defined by $w=z=0$ and, for any $\alpha$ satisfying $\alpha^{4}+\alpha-1=0, U$ contains the line $L_{\alpha}$ through the point $\left(-\alpha^{3}+\alpha^{2}-\alpha+1: 1: 0: 0\right)$ on $L$ and the point $\left(-\alpha^{3}-\alpha+1:-\alpha^{3}-\alpha^{2}+1: \alpha^{3}-\right.$ $\left.\alpha^{2}+\alpha+1: 1\right)$. The plane $H_{\alpha}$ containing $L$ and $L_{\alpha}$ is given by $z=\left(\alpha^{3}-\alpha^{2}+\alpha+1\right) w$, and one verifies that $U \cap H_{\alpha}$ consists of $L, L_{\alpha}$ and a smooth conic.

Let $U_{3} \subset \mathbb{P}_{\mathbb{F}_{3}}^{3}$ be the surface defined by equation (4.1), which is smooth. Let $\bar{U}_{3}$ be the base change of $U_{3}$ to an algebraic closure of $\mathbb{F}_{3}$, and $F: \bar{U}_{3} \rightarrow \bar{U}_{3}$ the geometric Frobenius morphism, defined by $(x, y, z, w) \mapsto\left(x^{3}, y^{3}, z^{3}, w^{3}\right)$. Choose a prime $\ell \neq 3$ and let $F^{*}$ be the endomorphism of $\mathrm{H}^{2}\left(\bar{U}_{3}, \mathbb{Q}_{\ell}(1)\right)$ induced by $F$. By [21, Proposition 6.2] the Picard rank of $\bar{U}$ is bounded above by that of $\bar{U}_{3}$, which in turn is at most the number of eigenvalues of $F^{*}$ that are roots of unity by [21, Corollary 6.4]. As in [21], we find the characteristic polynomial of $F^{*}$ by counting points on $U_{3}$. The results are shown in Table 1.

From the Lefschetz fixed point formula we find that the trace of the $n$th power of Frobenius acting on $\mathrm{H}_{\text {ett }}^{2}\left(\bar{U}_{3}, \mathbb{Q}_{\ell}\right)$ is equal to \# $U_{3}\left(\mathbb{F}_{3^{n}}\right)-3^{2 n}-1$. The trace on the Tate twist $\mathrm{H}_{\text {ét }}^{2}\left(\bar{U}_{3}, \mathbb{Q}_{\ell}(1)\right)$ is obtained by dividing by $3^{n}$, while on the subspace $V \subset$ $\mathrm{H}_{\text {ét }}^{2}\left(\bar{U}_{3}, \mathbb{Q}_{\ell}(1)\right)$ generated by $H, L$, and the four lines $L_{\alpha}$, the trace $t_{n}$ is equal to 6 if $n$ is a multiple of 4 , and equal to 2 if $n$ is not a multiple of 4 ; hence, on the 16-dimensional quotient $Q=\mathrm{H}_{\text {ett }}^{2}\left(\bar{U}_{3}, \mathbb{Q}_{\ell}(1)\right) / V$, the trace equals \# $U_{3}\left(\mathbb{F}_{3^{n}}\right) / 3^{n}-3^{n}-3^{-n}-t_{n}$. These traces are sums of powers of eigenvalues, and we use the Newton identities to compute the elementary symmetric polynomials in these eigenvalues, which are the coefficients of the characteristic polynomial $f$ of Frobenius acting on $Q$. This yields the first half of the coefficients of $f$, including the middle coefficient, which turns out to be non-zero. This implies that the sign in the functional equation $t^{16} f(1 / t)= \pm f(t)$ is +1 , so this functional equation determines $f$, which we calculate to be

$$
f=\frac{1}{3}\left(3 t^{16}+t^{14}+4 t^{13}+2 t^{10}-2 t^{8}+2 t^{6}+4 t^{3}+t^{2}+3\right) .
$$

As the characteristic polynomial of Frobenius acting on $V$ is $(t-1)^{2}\left(t^{4}-1\right)$, we find that the characteristic polynomial of Frobenius acting on $\mathrm{H}_{\text {ét }}^{2}\left(\bar{U}_{3}, \mathbb{Q}_{\ell}(1)\right)$ is equal to 
$(t-1)^{2}\left(t^{4}-1\right) f$. The polynomial $3 f \in \mathbb{Z}[t]$ is irreducible, primitive and not monic, so its roots are not roots of unity. Thus we obtain an upper bound of 6 for the Picard rank of $\bar{U}$.

Remark 4.8 We found $U$ by first fixing the lines $L$ and $L^{\prime}$. The space of rational quartic polynomials vanishing on $L$ and on the conjugates of $L^{\prime}$ is easily checked to have dimension 14. (This is the expected dimension. The space of quartic polynomials has dimension 35 and vanishing on a line is equivalent to vanishing on five points of the line and so imposes five conditions. However, if two lines meet in a point, that point gives the same condition for both lines. With four pairs of intersecting lines, we therefore expect the dimension to be $35-5 \times 5+4=14$.) We randomly chose elements of this space until we obtained one defining a smooth surface with good reduction at 3 and suitable characteristic polynomial of Frobenius.

Lemma 4.9 The surface $\bar{X}$ has exactly nine smooth rational curves.

Proof Let the $W_{i}$ be the components of the reducible fibres that meet $O$. In our basis, the known rational curves have classes $[O],\left[W_{i}\right],[E]-\left[W_{i}\right]$ for $1 \leqslant i \leqslant 4$. Suppose that there is another rational curve, of class $C=a[E]+b[O]+\sum_{i=1}^{4} c_{i}\left[W_{i}\right]$. It must have non-negative intersection with the known curves, which implies the inequalities

$$
a \geqslant b \geqslant 0, \quad c_{i} \leqslant 0, \quad b+c_{i} \geqslant 0, \quad 1 \leqslant i \leqslant 4 .
$$

Let $m=\min \left(\left\{c_{i}\right\}\right):$ then $a b \geqslant b^{2} \geqslant 4 m^{2}$. We thus find

$$
C^{2}=2 a b-2 \sum_{i=1}^{4} c_{i}^{2} \geqslant 2 b^{2}-8 m^{2} \geqslant 0,
$$

which contradicts the fact that $C^{2}=-2$.

Remark 4.10 The surface $\bar{U}$, on the other hand, has infinitely many smooth rational curves. Indeed, by [12, Chapter 11, Equation (4.5)], the Picard lattice of $\bar{U}$ has discriminant -144 . However, the list of Picard lattices of rank $\geqslant 6$ giving finite automorphism group in [14, Theorem 3.1] does not include any lattices of rank 6 and discriminant -144 . Hence Aut $\bar{U}$ is infinite. Let $\mathcal{C}$ be the union of $\{L\}$ and the set of components of the reducible fibres of $\pi_{L}$. By construction $\mathcal{C}$ spans a subgroup of Pic $\bar{U}$ of finite index (in fact, by computing the discriminant one checks that $\mathcal{C}$ generates $\operatorname{Pic} \bar{U}$ ). It follows that the stabilizer of $\mathcal{C}$ in Aut $\bar{U}$ is finite and hence that the orbit is infinite.

Remark 4.11 We now explain why we do not construct the Jacobian of $U$ directly as a smooth surface in a projective space.

If $H$ is an ample divisor class on a K3 surface $X$ with Pic $X \cong L_{N}$, then $H^{2} \geqslant 16$. To see this, let $H=a[E]+b[O]+\sum_{i=1}^{4} c_{i}\left[W_{i}\right]$ be such a class. Since $H$ is ample, all of $H \cdot[O], H \cdot\left[W_{i}\right], H \cdot\left([E]-\left[W_{i}\right]\right)$ must be positive: that is,

$$
a-2 b+\sum_{i=1}^{4} c_{i} \geqslant 1, \quad b-2 c_{i} \geqslant 1, \quad c_{i} \geqslant 1 .
$$


So $b \geqslant 3$ and $b+2 c_{i} \geqslant 5$. We thus find

$$
\begin{aligned}
H^{2} & =2 a b-2 b^{2}+2 \sum_{i=1}^{4} b c_{i}-2 \sum_{i=1}^{4} c_{i}^{2} \\
& \geqslant 2 b\left(2 b-\sum_{i=1}^{4} c_{i}+1\right)-2 b^{2}+2 \sum_{i=1}^{4} b c_{i}-2 \sum_{i=1}^{4} c_{i}^{2} \\
& =2 b^{2}+2 b-2 \sum_{i=1}^{4} c_{i}^{2} \\
& =2 b+\frac{\sum_{i=1}^{4}\left(b-2 c_{i}\right)\left(b+2 c_{i}\right)}{2} \\
& \geqslant 6+4 \cdot 1 \cdot 5 / 2=16 .
\end{aligned}
$$

Conversely, such a surface has an ample divisor class of self-intersection 16 . To see this, note that equality is attained in the above with $a=3, b=3, c_{i}=1$, and this gives an ample divisor class by [12, Corollary 8.1.7].

Thus an ample divisor class cannot give an embedding into $\mathbb{P}^{n}$ for $n<9$.

\subsection{Second example}

Now we give an example that is perhaps more surprising: a $\mathrm{K} 3$ surface $Y / \mathbb{Q}$ for which Aut $Y$ is finite even though, for all field extensions $L / \mathbb{Q}$, a K3 surface over $\overline{\mathbb{Q}}$ whose Picard lattice is isomorphic to $\operatorname{Pic} Y_{L}$ would have infinite automorphism group.

Definition 4.12 Let

$$
M=\left(\begin{array}{cc}
10 & 0 \\
0 & -4
\end{array}\right), \quad N=\left(\begin{array}{ccc}
10 & 0 & 0 \\
0 & -2 & 0 \\
0 & 0 & -2
\end{array}\right)
$$

and let $L_{M}, L_{N}$ be the lattices with Gram matrices $M, N$ respectively.

We will choose $Y$ to be a K3 surface whose Picard lattice over $\mathbb{Q}$ is isomorphic to $L_{M}$, while over $\overline{\mathbb{Q}}$, and indeed over a certain quadratic extension $K_{Y}$, the Picard lattice is isomorphic to $L_{N}$. The Galois group will act through the quotient $\mathbb{Z} / 2 \mathbb{Z}$ by exchanging the second and third generators.

Proposition 4.13 Let $Y$ be a $K 3$ surface in $\mathbb{P}_{\mathbb{Q}}^{4}$ given as the intersection of a quadric and a cubic, containing two disjoint Galois-conjugate conics $C_{1}, C_{2}$ defined over a quadratic field $K_{Y}$, and having Picard number 3 over $\overline{\mathbb{Q}}$. Then Pic $Y \cong L_{M}$ and $\operatorname{Pic} Y_{\overline{\mathbb{Q}}} \cong L_{N}$.

Proof Let $H$ be a hyperplane section. Then the divisors $H+C_{1}+C_{2}, C_{1}, C_{2}$ have the intersection matrix $N$, whose determinant is -40 . Thus we have an embedding 
$L_{N} \hookrightarrow$ Pic $Y_{\overline{\mathbb{Q}}}$ whose image has index 1 or 2 . If it is 2 , then either $[H]+\left[C_{1}\right]$ or $[H]+\left[C_{2}\right]$ can be divided by 2 . If $[H]+\left[C_{1}\right] \sim 2 D$, then, letting $\sigma$ be an extension of the non-trivial automorphism of the field of definition of $C_{1}$ to that of $D$, we have $[H]+\left[C_{2}\right] \sim 2 D^{\sigma}$, and so both classes can be divided by 2 and the index is a multiple of 4 , a contradiction. Similarly, $[H]+\left[C_{2}\right]$ cannot be divided by 2 . We conclude that the index is 1: that is, Pic $Y_{\overline{\mathbb{Q}}} \cong L_{N}$. There are Galois-invariant divisors of the classes $[H]+\left[C_{1}\right]+\left[C_{2}\right],\left[C_{1}\right]+\left[C_{2}\right]$, and these span the invariant subspace, so they generate Pic $Y$. Their intersection matrix is $M$.

Remark 4.14 The divisor class $[H]$ is very ample, because it is the hyperplane class on the smooth projective surface $Y$. The divisor class $D=[H]+\left[C_{1}\right]+\left[C_{2}\right]$ is not ample, but we will show that it is nef. Indeed, for any irreducible curve $C$ other than $C_{1}$ and $C_{2}$, the intersection numbers $H \cdot C, C_{1} \cdot C, C_{2} \cdot C$ are all non-negative, while $D \cdot\left[C_{1}\right]$ and $D \cdot\left[C_{2}\right]$ are both zero. In fact, one can show that $D$ is the hyperplane class for a model of $Y$ as a surface of degree 10 in $\mathbb{P}^{6}$ with two ordinary double points $\left(A_{1}\right.$ singularities).

Proposition 4.15 (1) The group $\mathrm{O}\left(L_{M}\right)$ is infinite. If $\alpha \in \mathrm{O}\left(L_{M}\right)$ has infinite order and $A$ is a normal subgroup of $\mathrm{O}\left(L_{M}\right)$ containing $\alpha$ then $\mathrm{O}\left(L_{M}\right) / A$ is finite.

(2) Let $V$ be a K3 surface over an algebraically closed field having Picard lattice isomorphic to $L_{M}$. Then Aut $V$ is infinite.

(3) Let $W$ be a K3 surface over an algebraically closed field having Picard lattice isomorphic to $L_{N}$. Then Aut $W$ is infinite.

Proof We first prove (1). To find $\mathrm{O}\left(L_{M}\right)$, we consider the quadratic form $10 x^{2}-4 y^{2}=$ $-N(2 y+\sqrt{10} x)$ associated to $M$. Its automorphism group is generated by the sign changes $(x, y) \mapsto(x,-y)$ and $(x, y) \mapsto(-x, y)$ and by multiplication by a generator of the group of totally positive units of $\mathrm{O}_{\mathbb{Q}(\sqrt{10})}$. (This generator is $19+6 \sqrt{10}$ and it takes $(x, y)$ to $(19 x+12 y, 30 x+19 y)$.) Thus $\mathrm{O}\left(L_{M}\right)$ has a subgroup of finite index isomorphic to $\mathbb{Z}$, so the quotient by any infinite normal subgroup is finite.

We continue with (2). Working mod 5 we see that Pic $V$ has no vectors of norm -2 , whence $V$ has no rational curves. Thus Aut $V$ coincides up to finite index with the infinite group $\mathrm{O}(\mathrm{Pic} V)=\mathrm{O}\left(L_{M}\right)$ : this follows from Theorem 1.1(2), because $\mathrm{W}(\mathrm{Pic} V)$ is trivial.

We now turn to (3). Let $D_{1}, D_{2}, D_{3}$ be divisors on $W$ whose intersection matrix is $N$, and let $D=D_{1}-2 D_{2}-D_{3}$. Since $D^{2}=0$ and $D$ is primitive, for some $\alpha \in \mathrm{O}\left(L_{N}\right)$ there is a genus-1 fibration $\pi$ with fibres of class $\alpha(D)$. There is no section of $\pi$ (indeed, no two curves on $W$ have odd intersection), but there is a 2 -section. The Jacobian $J$ of $\pi$ is a K3 surface of Picard number 3 [12, Corollary 11.4.7] on which the determinant of the intersection pairing is $(\operatorname{det} N) / 2^{2}=-10$ [12, Equation (11.4.5)]. As in Proposition 4.1, this shows that $J$ has no reducible fibres: the non-identity component of a reducible fibre would be orthogonal to the classes of both a fibre and the zero-section, and have self-intersection -2 , which is incompatible with the required determinant. The Shioda-Tate formula then shows that the Mordell-Weil group of $J$ has rank 1 . This Mordell-Weil group acts faithfully on $W$, so it follows as in Proposition 4.1 that Aut $W$ is infinite. 
Proposition 4.15 states that any K3 surface over an algebraically closed field having Picard lattice isomorphic to either $L_{M}$ or $L_{N}$ has infinite automorphism group. In contrast, the following proposition shows that a $\mathrm{K} 3$ surface over $\mathbb{Q}$ having Picard lattice isomorphic to $L_{M}$ or $L_{N}$ over any algebraic extension of $\mathbb{Q}$ may have finite automorphism group. Indeed, the last part of this section will be devoted to finding an example of such a surface.

Proposition 4.16 Let $Y$ be a K3 surface as in Proposition 4.13. Then Aut $Y$ is finite.

Proof Let $D_{1}=[H]+\left[C_{1}\right]+\left[C_{2}\right]$ and $D_{2}=\left[C_{1}\right]+\left[C_{2}\right]$, so that $D_{1}, D_{2}$ have the intersection matrix $M$. Since $C_{1}, C_{2}$ are disjoint conjugate rational curves, the product $r_{1}$ of the reflections in their classes is in our reflection group $R_{Y}$ (Definition 3.3). In the given basis, $r_{1}$ acts on Pic $Y$ with matrix

$$
A_{1}=\left(\begin{array}{cc}
1 & 0 \\
0 & -1
\end{array}\right) \text {. }
$$

We now show that there is a smooth rational curve in the class $F=6 D_{1}-9\left[C_{1}\right]-$ $10\left[C_{2}\right]$, defined over the same field $K_{Y}$ as the $C_{i}$. The class $F$ is effective, since it has self-intersection -2 and positive intersection with the very ample divisor class $[H]$. An irreducible curve of self-intersection -2 must be rational and smooth, so it suffices to show that $F$ has no non-trivial expression as a sum of effective classes.

Let $E$ be an effective divisor with $[E]=a D_{1}-b_{1}\left[C_{1}\right]-b_{2}\left[C_{2}\right]$. By Remark 4.14, $D_{1}$ is nef, so $0 \leqslant[E] \cdot D_{1}=10 a$, and therefore $a \geqslant 0$. Moreover, if $E$ is irreducible, then the inequality $E^{2} \geqslant-2$ yields $10 a^{2} \geqslant 2 b_{1}^{2}+2 b_{2}^{2}-2$, and if furthermore we have $a>0$, then the inequality $C_{i} \cdot E \geqslant 0$ gives $b_{i} \geqslant 0$.

We write the class $F$ as a sum $F=\sum_{i=1}^{s}\left[E_{i}\right]$ of classes of irreducible effective divisors $E_{1}, \ldots, E_{s}$, and we write $\left[E_{i}\right]=a_{i} D_{i}-b_{i, 1}\left[C_{1}\right]-b_{i, 2}\left[C_{2}\right]$. We saw above that $a_{i} \geqslant 0$ for each $i$, so from $\sum_{i} a_{i}=6$, we find $a_{i} \leqslant 6$ for each $i$. Furthermore, from $\sum_{i}\left(b_{i, 1}+b_{i, 2}-3 a_{i}\right)=9+10-3 \cdot 6=1>0$, we conclude that there is an $i$ with $b_{i, 1}+b_{i, 2}>3 a_{i}$, and hence $b_{i, 1}+b_{i, 2} \geqslant 3 a_{i}+1$. Again from the above, we find for such $i$ that

$$
10 a_{i}^{2} \geqslant 2 b_{i, 1}^{2}+2 b_{i, 2}^{2}-2 \geqslant\left(b_{i, 1}+b_{i, 2}\right)^{2}-2 \geqslant\left(3 a_{i}+1\right)^{2}-2,
$$

which implies $a_{i} \geqslant 6$, so $a_{i}=6$. The left- and right-hand sides of the sequence of inequalities above then differ by only $1=360-359$, so we also find $b_{i, 1}+b_{i, 2}=$ $3 a_{i}+1=19$ (not -19 because $\left.b_{i, j} \geqslant 0\right)$ and $\left(2 b_{i, 1}^{2}+2 b_{i, 2}^{2}\right)-\left(b_{i, 1}+b_{i, 2}\right)^{2} \leqslant 1$. Equivalently, $\left(b_{i, 1}-b_{i, 2}\right)^{2} \leqslant 1$, which yields $\left[E_{i}\right]=F$ or $\left[E_{i}\right]=F^{\prime}=6 D_{1}-$ $10\left[C_{1}\right]-9\left[C_{2}\right]$. But $F-\left[E_{i}\right]$ is effective by definition, and $F-F^{\prime}=\left[C_{1}\right]-\left[C_{2}\right]$ is not effective (the intersection with the ample divisor $H$ is 0 ), so $\left[E_{i}\right]=F$ and $F$ is indeed the class of an irreducible curve, say $R$.

Let $\sigma$ be an automorphism of $\overline{\mathbb{Q}}$ that exchanges $C_{1}$ and $C_{2}$. Then the class $F^{\sigma}=$ $\left[R^{\sigma}\right]$ is $6 D_{1}-10\left[C_{1}\right]-9\left[C_{2}\right]$, and one checks immediately that $F \cdot F^{\sigma}=0$. The product $r_{2}$ of the reflections in $F$ and $F^{\sigma}$ has matrix 


$$
A_{2}=\left(\begin{array}{cc}
721 & 456 \\
-1140 & -721
\end{array}\right)
$$

with respect to the basis $\left(D_{1}, D_{2}\right)$ of Pic $Y$. Now, $r_{1} r_{2}$ is an element of $R_{Y}$ of infinite order. From Proposition 4.15 (1), it follows that the quotient $\mathrm{O}(\mathrm{Pic} Y) / R_{Y}$ is finite and hence that Aut $Y$ is finite.

Remark 4.17 Given equations for $Y$, it is quite practical to make the curve $R$ used in the proof explicit. For any variety $V \subset \mathbb{P}^{4}$, let $I(V)$ be the ideal in the homogeneous coordinate ring of $\mathbb{P}^{4}$ of polynomials that vanish on $V$. Then the dimension of the degree-6 part of $\left(\left(I(Y)+I\left(C_{1}\right)^{3}\right) \cap\left(I(Y)+I\left(C_{2}\right)^{4}\right)\right) / I(Y)$ is 1 ; let $f_{6}$ be a generator, so that the divisor cut out on $Y$ by $f_{6}$ is of the form $C+3 C_{1}+4 C_{2}$. Using Magma one checks that $C$ is indeed an irreducible curve of arithmetic genus 0 .

Remark 4.18 The numerical properties of $F$ follow from the fact that $(19,6)$ is a solution to the Pell equation $x^{2}-10 y^{2}=1$. More generally, let $k$ be even and suppose that $D_{1},\left[C_{1}\right],\left[C_{2}\right]$ are classes of pairwise disjoint divisors on a surface with $D_{1}^{2}=k$ and $C_{1}^{2}=C_{2}^{2}$, where $C_{1}$ and $C_{2}$ constitute a Galois orbit. Suppose further that $m^{2}-k n^{2}=1$ : clearly $m$ is odd. Let $D$ be the divisor class $n D_{1}-\left\lfloor\frac{m}{2}\right\rfloor\left[C_{1}\right]-\left\lceil\frac{m}{2}\right\rceil\left[C_{2}\right]$ and let $D^{\sigma}$ be its Galois conjugate. Then $D^{2}=k n^{2}-2\left(\frac{m-1}{2}\right)^{2}-2\left(\frac{m+1}{2}\right)^{2}=-2$ and $D \cdot D^{\sigma}=k n^{2}-4 \frac{(m-1)(m+1)}{4}=0$.

The remainder of this section will be devoted to constructing a K3 surface $Y$ satisfying the conditions of Proposition 4.13. The verification will be more complicated than that of Example 4.7, because the Picard number over $\overline{\mathbb{Q}}$ in this example is odd, so that the reduction map cannot induce an isomorphism of Pic $Y_{\overline{\mathbb{Q}}}$ to Pic $Y_{\overline{\mathbb{F}}_{p}}$. We use the following proposition.

Proposition 4.19 Let $Y$ be a smooth intersection of a quadric and a cubic in $\mathbb{P}^{4}(\mathbb{Q})$ containing two disjoint conics $C_{1}, C_{2}$ that are defined and conjugate over a quadratic field $K_{Y}$. Let $p \neq 2, q$ be primes such that:

(1) $Y$ has good reduction at $p$ and $q$;

(2) the reduction $Y_{\mathbb{F}_{p}}$ contains a line $L$ disjoint from the reductions of $C_{1}, C_{2}$, and its Picard group over $\overline{\mathbb{F}}_{p}$ has rank 4 ;

(3) the reduction $Y_{\mathbb{F}_{q}}$ base changed to $\overline{\mathbb{F}}_{q}$ contains no lines disjoint from the reductions of $C_{1}$ and $C_{2}$.

Then $Y_{\overline{\mathbb{Q}}}$ has Picard number 3 and hence $Y$ satisfies the conditions of Proposition 4.13 .

Remark 4.20 To speak of $Y_{\mathbb{F}_{p}}$ we first need to choose a model of $Y$ over $\mathbb{Z}$. When we give equations with integral coefficients for $Y$ over $\mathbb{Q}$, it is understood that the model over $\mathbb{Z}$ is defined by the same equations. In each case it may be checked that the ideal defining this model is generated by the intersection of the ideal defining $Y$ over $\mathbb{Q}$ with the ring of polynomials in the same variables with integral coefficients.

Proof Let $s_{p}$ be the specialization homomorphism $\operatorname{Pic} Y_{\overline{\mathbb{Q}}} \rightarrow \operatorname{Pic} Y_{\overline{\mathbb{F}}_{p}}$, which is injective by [21, Proposition 6.2]. Then by [8, Theorem 1.4] the cokernel of $s_{p}$ 
is torsion-free. Note that specialization preserves intersection numbers $[10$, Section 20.3]. If $s_{p}$ is surjective, the class of $L$ is in the image. The class $L_{0}$ with $s_{p}\left(L_{0}\right)=[L]$ has positive intersection with the hyperplane class and self-intersection -2 , so it is effective; its intersection with the hyperplane class is 1 , so it is the class of a line on $Y_{\overline{\mathbb{Q}}}$. We have $[L] \cdot s_{p}\left(C_{i}\right)=0$, so $L_{0} \cdot C_{i}=0$ and $s_{q}\left(L_{0}\right) \cdot s_{q}\left(C_{i}\right)=0$ as well. This contradicts hypothesis (3). It follows that rk Pic $Y_{\overline{\mathbb{Q}}}<\operatorname{rk} \operatorname{Pic} Y_{\overline{\mathbb{F}}_{p}}=4$. On the other hand, the hyperplane class in $\mathbb{P}^{4}$ and the classes of $C_{1}, C_{2}$ are independent, so rk Pic $Y_{\overline{\mathbb{Q}}} \geqslant 3$.

In our construction, we will use $p=3, q=5$. We will take the conics $C_{1}, C_{2}$ to be defined over $\mathbb{Q}(\sqrt{19})$, the smallest quadratic field in which both $p$ and $q$ split. Let $\rho=\sqrt{19}$, and denote the coordinates on $\mathbb{P}^{4}$ by $x_{0}, \ldots, x_{4}$. We choose the conic $C_{1}$ defined by

$$
\begin{aligned}
& (-2 \rho+2) x_{0}+x_{2}+(\rho-2) x_{3}+(-\rho-2) x_{4} \\
& =(2 \rho+1) x_{0}+(\rho+2) x_{1}+2 x_{2}+(\rho+2) x_{3}+(-\rho+2) x_{4} \\
& =(-\rho-2) x_{0}^{2}+(-2 \rho+1) x_{0} x_{1}+(-2 \rho-1) x_{1}^{2} \\
& \quad+(-\rho+2) x_{0} x_{2}+(-\rho+2) x_{1} x_{2}+(\rho-2) x_{2}^{2} \\
& \quad+(2 \rho+1) x_{0} x_{3}+(2 \rho-2) x_{1} x_{3}+(2 \rho+2) x_{2} x_{3}-2 x_{3}^{2} \\
& \quad+(\rho+2) x_{0} x_{4}+(-\rho+2) x_{1} x_{4}+(-\rho+1) x_{2} x_{4}-x_{3} x_{4}+2 x_{4}^{2}=0
\end{aligned}
$$

and let $C_{2}$ be its Galois conjugate. Next, let $C_{3,1}, C_{3,2}$ be the reductions of $C_{1}$ and $C_{2}$, respectively, at one of the two primes above 3 , and let $L$ be the line in $\mathbb{P}^{4}\left(\mathbb{F}_{3}\right)$ through $(1: 1: 2: 0: 1)$ and $(2: 1: 1: 1: 0)$.

Proposition 4.21 The surface $Y_{3}$ in $\mathbb{P}^{4}\left(\mathbb{F}_{3}\right)$ defined by $F_{3}=G_{3}=0$, where

$$
\begin{aligned}
F_{3}=2 x_{0} x_{1} & +x_{1} x_{2}+x_{2}^{2}+2 x_{0} x_{3}+x_{1} x_{3}+x_{3}^{2}+2 x_{0} x_{4}+x_{3} x_{4}+2 x_{4}^{2}, \\
G_{3}=2 x_{0} x_{1}^{2} & +2 x_{0}^{2} x_{2}+2 x_{0} x_{1} x_{2}+2 x_{1}^{2} x_{2}+2 x_{0} x_{2}^{2}+2 x_{1} x_{2}^{2}+2 x_{2}^{3} \\
& +2 x_{0} x_{1} x_{3}+x_{1}^{2} x_{3}+2 x_{0} x_{2} x_{3}+2 x_{1} x_{2} x_{3}+2 x_{0} x_{3}^{2}+2 x_{2} x_{3}^{2} \\
& +2 x_{3}^{3}+x_{0}^{2} x_{4}+2 x_{1}^{2} x_{4}+2 x_{0} x_{2} x_{4}+x_{2}^{2} x_{4}+2 x_{0} x_{3} x_{4} \\
& +2 x_{2} x_{3} x_{4}+x_{3}^{2} x_{4}+2 x_{0} x_{4}^{2}+x_{1} x_{4}^{2}+x_{2} x_{4}^{2}+x_{4}^{3},
\end{aligned}
$$

is smooth, contains $L, C_{3,1}$, and $C_{3,2}$, and has Picard number 4.

Proof The verification that $Y_{3}$ is smooth and contains the given curves is routine. To see that $Y_{3}$ has Picard number 4, we proceed as in Example 4.7. In order to count the number of points on $Y_{3}$ over $\mathbb{F}_{3^{n}}$ for $1 \leqslant n \leqslant 9$, we write $Y_{3}$ as an elliptic surface using the fibration of Picard class $H-C_{3,1}$, for which $L$ is a section, and we sum the number of points of the fibres. We obtain point counts as in Table 2.

As in Example 4.7, we find that the trace of the $n$th power of Frobenius acting on $\mathrm{H}_{\text {ét }}^{2}\left(\bar{Y}_{3}, \mathbb{Q}_{\ell}(1)\right)$ is equal to \# $Y_{3}\left(\mathbb{F}_{3^{n}}\right) / 3^{n}-3^{n}-3^{-n}$, while on the subspace $V \subset$ $\mathrm{H}_{\text {et }}^{2}\left(\bar{Y}_{3}, \mathbb{Q}_{\ell}(1)\right)$ generated by $H, C_{3,1}, C_{3,2}$, and $L$, the trace is equal to 4 ; hence, on 
Table 2 Point counts on the surface $Y_{3}$

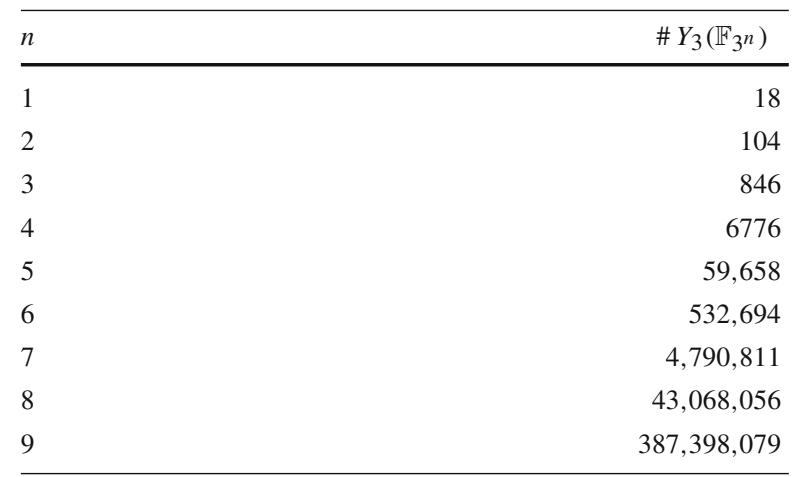

the 18-dimensional quotient $Q=\mathrm{H}_{\text {ét }}^{2}\left(\bar{Y}_{3}, \mathbb{Q}_{\ell}(1)\right) / V$, we calculate the trace to be $\# Y_{3}\left(\mathbb{F}_{3^{n}}\right) / 3^{n}-3^{n}-3^{-n}-4$. Again, these traces, together with the Newton identities, determine the coefficients of the characteristic polynomial $f$ of Frobenius acting on $Q$. The point counts in Table 2 yield the first half of the coefficients of $f$, including the middle coefficient, which turns out to be non-zero. This implies that the sign in the functional equation $t^{18} f(1 / t)= \pm f(t)$ is +1 , so this functional equation determines $f$, which we calculate to be

$$
\begin{aligned}
f=\frac{1}{3}\left(3 t^{18}-4 t^{17}+5 t^{16}-4 t^{15}+4 t^{14}-4 t^{13}+5 t^{12}-5 t^{11}+5 t^{10}\right. \\
\left.-6 t^{9}+5 t^{8}-5 t^{7}+5 t^{6}-4 t^{5}+4 t^{4}-4 t^{3}+5 t^{2}-4 t+3\right) .
\end{aligned}
$$

As the characteristic polynomial of Frobenius acting on $V$ is $(t-1)^{4}$, we find that the characteristic polynomial of Frobenius acting on $\mathrm{H}_{\text {et }}^{2}\left(\bar{Y}_{3}, \mathbb{Q}_{\ell}(1)\right)$ is $(t-1)^{4} f$. The polynomial $3 f$ is irreducible, primitive and not monic, so its roots are not roots of unity. Thus we obtain an upper bound of 4 for the Picard rank of $\bar{Y}_{3}$. The rank is equal to 4 as the Picard group contains the linearly independent classes of $H, L, C_{3,1}$, and $C_{3,2}$.

Now let $C_{5,1}, C_{5,2}$ be the reductions of $C_{1}$ and $C_{2}$, respectively, at one of the two primes above 5 .

Proposition 4.22 Let $Y_{5}$ be the surface in $\mathbb{P}_{\mathbb{F}_{5}}^{4}$ defined by $F_{5}=G_{5}=0$, where

$$
\begin{aligned}
F_{5}=2 x_{0}^{2} & +x_{0} x_{1}+3 x_{1}^{2}+2 x_{0} x_{2}+2 x_{2}^{2}+2 x_{1} x_{3} \\
& +2 x_{2} x_{3}+3 x_{3}^{2}+3 x_{0} x_{4}+2 x_{2} x_{4} \\
G_{5}=x_{0}^{2} x_{1} & +3 x_{0} x_{1}^{2}+2 x_{1}^{3}+4 x_{0} x_{1} x_{2}+x_{1}^{2} x_{2}+2 x_{1} x_{2}^{2} \\
& +3 x_{2}^{3}+2 x_{0}^{2} x_{3}+4 x_{1}^{2} x_{3}+2 x_{0} x_{3}^{2}+2 x_{1} x_{3}^{2}+2 x_{2} x_{3}^{2} \\
& +2 x_{3}^{3}+x_{0} x_{1} x_{4}+4 x_{1}^{2} x_{4}+x_{0} x_{2} x_{4}+2 x_{2}^{2} x_{4}+3 x_{1} x_{3} x_{4} \\
& +2 x_{2} x_{3} x_{4}+3 x_{3}^{2} x_{4}+3 x_{0} x_{4}^{2}+2 x_{1} x_{4}^{2}+3 x_{2} x_{4}^{2}+x_{3} x_{4}^{2} .
\end{aligned}
$$


Then $Y_{5}$ is smooth. It contains $C_{5,1}$ and $C_{5,2}$, but no lines over $\overline{\mathbb{F}}_{5}$ that are disjoint from $C_{5,1}$.

Proof Again, it is easy to check that $Y_{5}$ is smooth and that the $C_{5, i}$ are on $Y_{5}$. It is also easy to verify directly that $Y_{5}$ contains no lines defined over $\mathbb{F}_{5}$ by checking that no line through two distinct $\mathbb{F}_{5}$-points of $Y_{5}$ is contained in $Y_{5}$; however, this is not sufficient. Suppose that $L$ is a line contained in $Y_{5}$ and disjoint from $C_{5,1}$. We embed $Y_{5}$ in $\mathbb{P}_{\mathbb{F}_{5}}^{5}$ by the divisor class $H+\left[C_{5,1}\right]$, where $H$ is the hyperplane class in $\mathbb{P}_{\mathbb{F}_{5}}^{4}$. In this embedding, $L$ is still a line, because $L \cdot\left(H+\left[C_{5,1}\right]\right)=1$.

The image is a surface $T_{5}$ defined by three quadrics $Q_{1}, Q_{2}, Q_{3}$. For each $Q_{i}$, let $Z_{i}$ be the subscheme of $\mathbb{P}^{5} \times \mathbb{P}^{5}$ consisting of pairs of points $P, P^{\prime}$ for which $P=P^{\prime}$ or the line joining $P$ to $P^{\prime}$ is on the variety defined by $Q_{i}=0$. The image of $Z_{i}$ in $\mathbb{P}^{14}$ under the Plücker embedding is isomorphic to the Fano scheme of lines on $Q_{i}$. It is easy to check in Magma that the intersection of the images of the $Z_{i}$ in $\mathbb{P}^{14}$ is empty, which implies that there are no lines on $T_{5}$ even over $\overline{\mathbb{F}}_{5}$.

Remark 4.23 In fact, we claim that $Y_{5}$ contains no lines at all. Indeed, a calculation similar to the one in the proof of Proposition 4.22 shows that there are also no lines on $Y_{5}$ disjoint from $C_{5,2}$. To finish the proof of the claim, it suffices to show that there are no lines that meet both $C_{5,1}$ and $C_{5,2}$. Let $Z_{2}$ be the closure of the subset of $\mathbb{P}_{\mathbb{F}_{5}}^{4} \times \mathbb{P}_{\mathbb{F}_{5}}^{4}$ consisting of all pairs of points $(x, y)$ such that $x \neq y$ and the line through $x$ and $y$ lies on the hypersurface defined by $F_{5}$. Define $Z_{3}$ similarly for $G_{5}$. Since $C_{5,1}$ and $C_{5,2}$ are disjoint, the intersection $Z_{2} \cap Z_{3} \cap\left(C_{5,1} \times C_{5,2}\right)$ consists of all pairs $(x, y)$ with $x \in C_{5,1}$ and $y \in C_{5,2}$ for which there is a line on $Y_{5}$ through $x$ and $y$. We then check in Magma that this intersection is empty. This is done in our Magma scripts [5].

In principle one can prove directly that $Y_{5}$ has no lines by checking that the images of $Z_{2}$ and $Z_{3}$ under the Plücker embedding in $\mathbb{P}^{9}$ are disjoint, but this calculation is very slow.

It remains to show that there exists a surface $Y \subset \mathbb{P}_{\mathbb{Q}}^{4}$ containing the conics $C_{1}, C_{2}$ and lifting the surfaces $Y_{3}, Y_{5}$ described above. Denote by $V_{2}\left(C_{1} \cup C_{2}, \mathbb{Q}\right)$ the vector space of homogeneous forms of degree 2 , with coefficients in $\mathbb{Q}$, vanishing on $C_{1} \cup C_{2}$. One easily computes that $V_{2}\left(C_{1} \cup C_{2}, \mathbb{Q}\right)$ has dimension 5 ; let $q_{1}, \ldots, q_{5}$ be a $\mathbb{Z}$-basis for the free abelian group $V_{2}\left(C_{1} \cup C_{2}, \mathbb{Z}\right)$ of forms having integer coefficients. One also easily computes that the analogous spaces $V_{2}\left(C_{3,1} \cup C_{3,2}, \mathbb{F}_{3}\right)$ and $V_{2}\left(C_{5,1} \cup C_{5,2}, \mathbb{F}_{5}\right)$ both have dimension 5 over $\mathbb{F}_{3}$ and $\mathbb{F}_{5}$ respectively, which implies that the images of $q_{1}, \ldots, q_{5}$ span these spaces. Now the Chinese remainder theorem guarantees the existence of a quadratic form $F \in V_{2}\left(C_{1} \cup C_{2}, \mathbb{Z}\right)$ satisfying both $F \equiv F_{3}(\bmod 3)$ and $F \equiv F_{5}(\bmod 5)$. Similarly, the analogous spaces of cubic forms vanishing on $C_{1} \cup C_{2}$ are all of dimension 21, which implies the existence of a cubic form $G$ satisfying $G \equiv G_{3}(\bmod 3)$ and $G \equiv G_{5}(\bmod 5)$. Remark 4.24 below shows that one can avoid explicit computations to calculate these dimensions. The surface $Y$ given by $F=G=0$ reduces modulo 3 and 5 to $Y_{3}$ and $Y_{5}$, respectively.

Remark 4.24 Let $k$ be any field. Let $C_{1}, C_{2} \subset \mathbb{P}_{k}^{4}$ be two conics; suppose that the two planes spanned by $C_{1}, C_{2}$ respectively meet only in one point $P$, and suppose that $P$ does not lie on either $C_{1}$ or $C_{2}$. Then the space of forms of degree $d$ vanishing on $C_{1}$ and $C_{2}$ has dimension 


$$
\begin{aligned}
4\left(\begin{array}{c}
d+2 \\
4
\end{array}\right)-4\left(\begin{array}{c}
d+1 \\
4
\end{array}\right) & +\left(\begin{array}{l}
d \\
4
\end{array}\right)+2\left(\begin{array}{l}
d \\
2
\end{array}\right)-1 \\
& =\frac{1}{24} d(d-1)\left(d^{2}+11 d+46\right)-1
\end{aligned}
$$

\subsection{Finite automorphism group for a family of diagonal surfaces}

In this example, for which we summarize the calculations in a Magma script [5], we consider a quartic surface $Z$ over a field $k$ of characteristic 0 defined by

$$
x^{4}-y^{4}=c\left(z^{4}-w^{4}\right)
$$

for some non-zero $c \in k$. [22, Conjecture 2.5] states that if $k$ is the field $\mathbb{Q}$ of rational numbers, then for every non-zero $c \in \mathbb{Q}$, the set of rational points on the associated surface $Z$ is dense. The 48 lines on a surface in this family are defined over the field $k\left(\zeta_{8}, \sqrt[4]{c}\right)$. We assume that $k, c$ are such that this is a Galois extension of $k$ of degree 16: with $k=\mathbb{Q}$, this is equivalent to $|c|$ being neither a square nor twice a square. (This Galois action is case A45 of [4, Appendix A].) We will prove under this assumption that Aut $Z$ is finite. The argument will proceed by the following steps.

1. Determination of Pic $Z$, and comparison to the Picard lattice of a K3 surface $V$ with finite automorphism group over $\bar{k}$.

2. Determination of $\mathrm{O}(\operatorname{Pic} V)$ and $\mathrm{O}(\operatorname{Pic} Z)$ up to finite index.

3. Construction of elements of $R_{Z}$ and verification that $\mathrm{O}(\mathrm{Pic} Z) / R_{Z}$ is finite.

Remark 4.25 If the characteristic of $k$ is congruent to $1 \bmod 4$, then $k$ contains the 4th roots of 1 and this Galois action is not possible. If it is $3 \bmod 4$, then $Z$ is a supersingular surface and $Z_{\bar{k}}$ has Picard number 22 , so the argument given here does not apply. If $k$ has characteristic 2, then of course $Z$ is not a smooth surface.

Let $L_{N}$ be the lattice with Gram matrix

$$
N=\left(\begin{array}{ll|l}
0 & 1 & \\
1 & 0 & \\
\hline & & -2 I_{4}
\end{array}\right),
$$

as in Section 4.1.

Lemma 4.26 There is a sublattice of $L_{N}$ of index 4 that is isomorphic to Pic $Z$.

Proof The Picard group of $\bar{Z}$ is generated by the classes of the 48 lines. (See [17, Sect. 6.1] for a history of proofs of this fact.) One easily verifies that the subspace fixed by the action of Galois is of rank 6 and that the intersection form has discriminant -256 . Since the surface has rational points such as $(1,1,0,0)$, the fixed subspace of the Picard group of $\bar{Z}$ is in fact the Picard group of $Z$.

Let $\Lambda$ be the lattice obtained from Pic $Z$ by adjoining $v / 2$ for each $v$ in

$$
\{v \in \operatorname{Pic} Z: 8 \mid(v, v) \text { and } 2 \mid(v, w) \text { for all } w \in \operatorname{Pic} Z\} \text {. }
$$


Then $\Lambda$ contains Pic $Z$ with index 4 and hence has discriminant -16 . It is verified in [5] that $L_{N}$ and $\Lambda$ are isomorphic.

We now proceed to step 2, determining $\mathrm{O}\left(L_{N}\right)$. Let $V$ be a K3 surface over $\bar{k}$ with Picard lattice $L_{N}$, such as those constructed in Proposition 4.6. We choose a basis $e_{1}, \ldots, e_{6}$ for $L_{N} \cong \operatorname{Pic} V$ to consist of the classes $E, E+O, C_{1}, C_{2}, C_{3}, C_{4}$, where $E$ is the class of an elliptic fibration with zero section $O$ and the $C_{i}$ are the components of the reducible fibres that do not meet $O$. In this basis, the Gram matrix of Pic $V$ is as above. Let $G \subset \mathrm{O}\left(L_{N}\right)$ be the image of the symmetric group $\mathcal{S}_{4}$ by the homomorphism taking a permutation $\pi$ to the automorphism fixing $E$ and $E+O$ and taking $C_{i}$ to $C_{\pi(i)}$. Let $A_{L_{N}}$ be the discriminant group of $L_{N}$ with its discriminant form, a quadratic form with values in $\mathbb{Q} / 2 \mathbb{Z}[12$, Chapter 14].

Lemma $4.27 A_{L_{N}}$ is generated by the classes of $e_{i} / 2$ for $3 \leqslant i \leqslant 6$. The discriminant form takes $\sum_{i=3}^{6} a_{i} e_{i} / 2$ to $-\sum_{i=3}^{6} a_{i}^{2} / 2 \bmod 2$, and the natural map $G \rightarrow$ Aut $A_{L_{N}}$ is an isomorphism.

Proof The $e_{i} / 2$ for $3 \leqslant i \leqslant 6$ belong to $L_{N}^{*}$ and they generate a group that contains $L_{N}$ with index 16 . Since $L_{N}$ has discriminant 16 , they generate $L_{N}^{*}$ and their classes generate $A_{L_{N}}$. The quadratic form takes $\sum_{i=3}^{6} a_{i} e_{i} / 2$ to $\sum_{i=3}^{6} a_{i}^{2} e_{i}^{2} / 4 \bmod 2=$ $-\sum_{i=3}^{6} a_{i}^{2} / 2$. It follows that the only elements of $A_{L_{N}}$ taken to $-1 / 2$ by the quadratic form are the basis vectors, so every automorphism of $A_{L_{N}}$ must permute these. Conversely it is clear that every permutation of the basis vectors extends to an automorphism of $A_{L_{N}}$ and that this automorphism is the image of a unique element of $G$.

Recall [12, Definition 15.1.1] that an automorphism of $V$ is symplectic if it acts trivially on $\mathrm{H}^{0}\left(V, \Omega_{V}^{2}\right)$.

Proposition 4.28 The group Aut $V$ is finite cyclic and the subgroup Aut $_{\mathrm{s}} V$ of symplectic automorphisms of $V$ is trivial.

Proof By Lemma 4.9, there are exactly nine smooth rational curves on $V$. Every automorphism of $V$ must preserve the configuration of these curves. Exactly one of them, the section of the elliptic fibration, intersects four of the others. Hence, the only automorphisms of the configuration are those that permute the reducible fibres. Since the nine curves generate Pic $V$, we conclude that every automorphism of $V$ acts on Pic $V$ by elements of $G$. Now, a symplectic automorphism $\alpha$ of a K3 surface $V$ in characteristic 0 acts as the identity on the transcendental lattice in $\mathrm{H}^{2}(V, \mathbb{Z})[12$, Remark 15.1.2] and hence on its discriminant group, so in addition it acts trivially on the discriminant group of Pic $V$ by [12, Lemma 14.2.5]. Since $\alpha$ acts on Pic $V$ through $G$, combining this with the last lemma shows that $\alpha$ acts trivially on Pic $V$. It follows that $\alpha$ acts trivially on $\mathrm{H}^{2}(V, \mathbb{Z})$; it is therefore the identity by [12, Corollary 15.1.6].

Since we are in characteristic 0 , there is an exact sequence [12, (15.1.3)]

$$
1 \rightarrow \text { Aut }_{\mathrm{s}} V \rightarrow \text { Aut } V \rightarrow \mu_{m} \rightarrow 1
$$


for some $m$, where Aut $V$ is the group of symplectic automorphisms that we have just shown to be trivial. It follows that Aut $V$ is finite cyclic.

Remark 4.29 There is always an automorphism of order 2 of $V$ given by negation of the elliptic fibration with respect to the unique section. For very general $V$ the order of Aut $V$ is 2, but it might be possible for Aut $V$ to be of order $m=4,6$, or 8 if there is an automorphism that permutes the reducible fibres of the fibration non-trivially. In this case the field of definition of Pic $V$ would contain $\mu_{m}$, which does not happen generically.

By [6, Proposition 5.10], we know that $\mathrm{O}\left(L_{N}\right)$ is generated by the automorphisms of the ample cone and the reflections in the classes of rational curves, together with -1 ; the automorphisms of the ample cone are just the $G$ of Lemma 4.27. Since $G$ is generated by two elements and has three orbits on the set of rational curves, we have a set of six generators for $\mathrm{O}\left(L_{N}\right)$. To pass to $\mathrm{O}(\mathrm{Pic} Z)$, we use a simple lemma.

Lemma 4.30 Let $\Lambda$ be a lattice with sublattices $\Lambda_{1}, \Lambda_{2}$ with $\left[\Lambda_{1}: \Lambda_{1} \cap \Lambda_{2}\right]$ finite. Then the stabilizer of $\Lambda_{1} \cap \Lambda_{2}$ in $\mathrm{O}\left(\Lambda_{1}\right)$ has finite index. If in addition $\Lambda_{1}, \Lambda_{2}$ have equal rank, then $\mathrm{O}\left(\Lambda_{1}\right) \cap \mathrm{O}\left(\Lambda_{2}\right)$ has finite index in $\mathrm{O}\left(\Lambda_{1}\right)$ and $\mathrm{O}\left(\Lambda_{2}\right)$, where the $\mathrm{O}\left(\Lambda_{i}\right)$ are regarded as subgroups of $\mathrm{O}\left(\Lambda_{1} \otimes \mathbb{Q}\right)$.

Proof There are only finitely many sublattices of $\Lambda_{1}$ of index $\left[\Lambda_{1}: \Lambda_{1} \cap \Lambda_{2}\right]$, so the stabilizer is of finite index. If $\left[\Lambda_{1}: \Lambda_{1} \cap \Lambda_{2}\right]$ is finite and $\Lambda_{1}, \Lambda_{2}$ have equal rank then $\left[\Lambda_{2}: \Lambda_{1} \cap \Lambda_{2}\right]$ is finite as well and $\mathrm{O}\left(\Lambda_{1}\right) \cap \mathrm{O}\left(\Lambda_{2}\right)$ is the stabilizer of $\Lambda_{1} \cap \Lambda_{2}$ in $\mathrm{O}\left(\Lambda_{i}\right)$ for $i \in\{1,2\}$.

Remark 4.31 The group generated by $\mathrm{O}\left(\Lambda_{1}\right)$ and $\mathrm{O}\left(\Lambda_{2}\right)$ usually does not contain $\mathrm{O}\left(\Lambda_{1}\right)$ with finite index.

Using Magma [3] to compute the permutation representation of $\mathrm{O}\left(L_{N}\right)$ on sublattices with quotient $(\mathbb{Z} / 2 \mathbb{Z})^{2}$, we find a set of 30 generators for $\mathrm{O}\left(L_{N}\right) \cap \mathrm{O}(\mathrm{Pic} Z)$. However, some of these are products of others, so we easily reduce to a set of nine generators. We now perform step 3, the construction of elements of $R_{Z}$.

Proposition 4.32 There are 14 Galois orbits of lines and eight of conics on $\bar{Z}$ that give finite Coxeter groups.

Proof We list the conics by observing that their Picard classes are of the form $H / 2+M$, where $M \in \frac{1}{2} \operatorname{Pic} \bar{Z}$ is such that $H \cdot M=0$ and $M^{2}=-3$. Since $H^{\perp} \subset \frac{1}{2} \mathrm{Pic} \bar{Z}$ is a definite lattice, the set of $M$ with these properties is finite. Such a class is represented by a conic if and only if it is integral and not the sum of two classes of lines, both of which are easily checked. We finish the proof by referring to Proposition 3.6 for the configurations of curves that give finite Coxeter groups.

Remark 4.33 It appears that there are no further generators of $R_{Z}$.

Theorem 4.34 Aut $Z$ is finite. 
Proof We use Proposition 3.10. To do so, we search for relations among the generators of $\mathrm{O}\left(L_{N}\right) \cap \mathrm{O}(\mathrm{Pic} Z)$ and the 22 elements of $R_{Z}$ that we have found, simply by checking which products of up to five generators of $\mathrm{O}\left(L_{N}\right) \cap \mathrm{O}(\mathrm{Pic} Z)$ and up to four of these together with the generators of $R_{Z}$ give the identity matrix. Magma quickly verifies that these relations are sufficient to show that $\left\langle\mathrm{O}\left(L_{N}\right) \cap \mathrm{O}(\mathrm{Pic} Z), R_{Z}\right\rangle / R_{Z}$ is finite, from which we conclude by Proposition 3.10 that Aut $Z$ is finite.

Corollary 4.35 There are finitely many classes of geometrically irreducible curves on $Z$ of each genus.

Proof This follows by combining the theorem with Corollary 3.16.

Remark 4.36 We conclude that in order to prove that the rational points of $Z$ are Zariski-dense, we do not have an infinite automorphism group Aut $Z$ to our disposal. In particular, we will not be able to find an elliptic fibration on $Z$ with a section of infinite order, as translation by such a section would be an automorphism of infinite order.

Acknowledgements We thank the referee, whose thorough report helped us improve the exposition and correct a number of errors in the paper.

Open Access This article is distributed under the terms of the Creative Commons Attribution 4.0 International License (http://creativecommons.org/licenses/by/4.0/), which permits unrestricted use, distribution, and reproduction in any medium, provided you give appropriate credit to the original author(s) and the source, provide a link to the Creative Commons license, and indicate if changes were made.

\section{References}

1. Ash, A., Mumford, D., Rapoport, M., Tai, Y.-S.: Smooth Compactifications of Locally Symmetric Varieties, 2nd edn. Cambridge Mathematical Library. Cambridge University Press, Cambridge (2010). With the collaboration of Peter Scholze

2. Björner, A., Brenti, F.: Combinatorics of Coxeter Groups. Graduate Texts in Mathematics, vol. 231. Springer, New York (2005)

3. Bosma, W., Cannon, J., Playoust, C.: The Magma algebra system. I. The user language. J. Symbolic Comput. 24(3-4), 235-265 (1997)

4. Bright, M.: Computations on Diagonal Quartic Surfaces. Ph.D. thesis, University of Cambridge (2002)

5. Bright, M., Logan, A., van Luijk, R.: Magma script. Available for download as an attachment to this article

6. Dolgachev, I.V.: Reflection groups in algebraic geometry. Bull. Amer. Math. Soc. 45(1), 1-60 (2008)

7. Eichler, M.: Quadratische Formen und Orthogonale Gruppen. Die Grundlehren der Mathematischen Wissenschaften in Einzeldarstellungen mit Besonderer Berücksichtigung der Anwendungsgebiete. Band LXIII. Springer, Berlin (1952)

8. Elsenhans, A.S., Jahnel, J.: The Picard group of a $K 3$ surface and its reduction modulo $p$. Algebra Number Theory 5(8), 1027-1040 (2011)

9. Fantechi, B., Göttsche, L., Illusie, L., Kleiman, S.L., Nitsure, N., Vistoli, A.: Fundamental Algebraic Geometry. Mathematical Surveys and Monographs, vol. 123. American Mathematical Society, Providence (2005). Grothendieck's FGA explained

10. Fulton, W.: Intersection Theory. Ergebnisse der Mathematik und ihrer Grenzgebiete. 3. Folge. A, vol. 2, 2nd edn. Springer, Berlin (1998)

11. Geck, M., Iancu, L.: Coxeter groups and automorphisms (2014). arXiv:1412.5428

12. Huybrechts, D.: Lectures on K3 Surfaces. Cambridge Studies in Advanced Mathematics, vol. 158. Cambridge University Press, Cambridge (2016) 
13. Lieblich, M., Maulik, D.: A note on the cone conjecture for K3 surfaces in positive characteristic (2011). arXiv:1102.3377

14. Nikulin, V.V.: Elliptic fibrations on K3 surfaces. Proc. Edinburgh Math. Soc. 57(1), 253-267 (2014)

15. Nikulin, V.V.: Integer symmetric bilinear forms and some of their geometric applications. Izv. Akad. Nauk SSSR Ser. Mat. 43(1), 111-177 (1979)

16. Rockafellar, R.T.: Convex Analysis. Princeton Landmarks in Mathematics. Princeton University Press, Princeton (1997)

17. Schütt, M., Shioda, T., van Luijk, R.: Lines on Fermat surfaces. J. Number Theory 130(9), 1939-1963 (2010)

18. Shimada, I.: An algorithm to compute automorphism groups of $K 3$ surfaces and an application to singular K3 surfaces. Int. Math. Res. Not. IMRN 2015(22), 11961-12014 (2015)

19. Silverman, J.H.: Advanced Topics in the Arithmetic of Elliptic Curves. Graduate Texts in Mathematics, vol. 151. Springer, New York (1994)

20. Sterk, H.: Finiteness results for algebraic K3 surfaces. Math. Z. 189(4), 507-513 (1985)

21. van Luijk, R.: An elliptic $K 3$ surface associated to Heron triangles. J. Number Theory 123(1), 92-119 (2007)

22. van Luijk, R.: Density of rational points on elliptic surfaces. Acta Arith. 156(2), 189-199 (2012)

Publisher's Note Springer Nature remains neutral with regard to jurisdictional claims in published maps and institutional affiliations. 